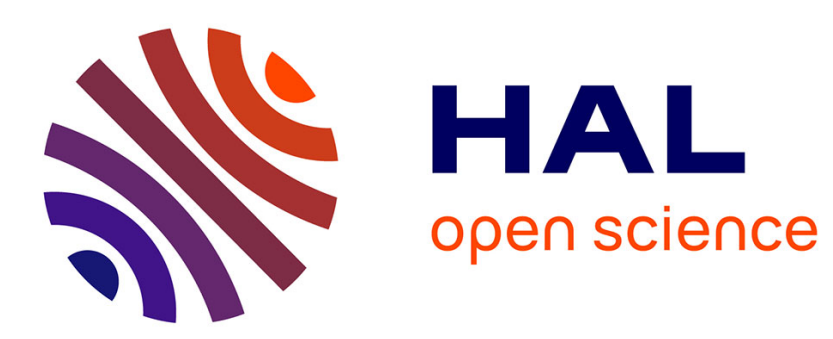

\title{
A differential geometric setting for dynamic equivalence and dynamic linearization
}

Jean-Baptiste Pomet

\section{To cite this version:}

Jean-Baptiste Pomet. A differential geometric setting for dynamic equivalence and dynamic linearization. Banach Center Publications, 1995, 32, pp.319 - 339. 10.4064/-32-1-319-339 . hal-01501078

\section{HAL Id: hal-01501078 \\ https://hal.inria.fr/hal-01501078}

Submitted on 3 Apr 2017

HAL is a multi-disciplinary open access archive for the deposit and dissemination of scientific research documents, whether they are published or not. The documents may come from teaching and research institutions in France or abroad, or from public or private research centers.
L'archive ouverte pluridisciplinaire HAL, est destinée au dépôt et à la diffusion de documents scientifiques de niveau recherche, publiés ou non, émanant des établissements d'enseignement et de recherche français ou étrangers, des laboratoires publics ou privés. 
A differential geometric setting for dynamic equivalence and dynamic linearization

J.-B. Pomet ${ }^{3}$

\title{
Infinitesimal Brunovský form for nonlinear systems, with applications to dynamic linearization \\ E. Aranda-Bricaire ${ }^{1}$ \\ C. H. $\mathrm{Moog}^{2}$ \\ J.-B. Pomet ${ }^{3}$
}

\begin{abstract}
These two articles have appeared in the volume:
Geometry in Nonlinear Control and Differential Inclusions, B. Jakubczyk et al. eds, Banach Center Publications, Vol. 32, Polish Academy of Sc., Warszawa, 1995, pp. 319-339 and pp. 19-33.
\end{abstract}

They are gathered here as one text in two chapters. Their contents is unchanged except that

- a few redundancies have been eliminated,

- the two lists of bibliographical references have been merged into one, and

- cross-referencing between the two papers has been improved.

February, 2002.

\footnotetext{
${ }^{1}$ Seccion de Mecatronica, Dep. de Ingenieria Electrica, CINVESTAV-IPN, Apartado Postal 14-740, 07000 Mexico, D.F., MEXICO, earanda@mail.cinvestav.mx.

${ }^{2}$ IRCCyN, B.P. 92101, 1, rue de la Noë, 44321 Nantes cedex 03, France, Claude.Moog@irccyn.ec-nantes.fr

${ }^{3}$ I.N.R.I.A., B.P. 93, 06902 Sophia Antipolis cédex, France, pomet@sophia.inria.fr.
} 


\section{Contents}

I A differential geometric setting for dynamic equivalence and dynamic linearization

I.1 Introduction . . . . . . . . . . . . . . . . . . . . . . 3

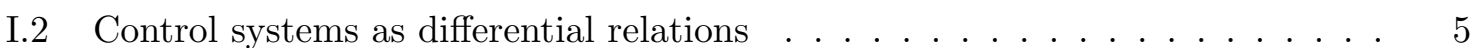

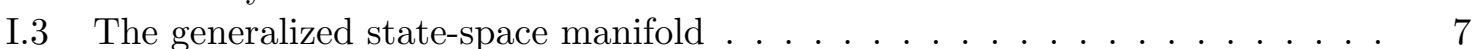

I.3.1 The manifold, functions and mappings . . . . . . . . . . . . . . 7

I.3.2 Vector fields and differential forms . . . . . . . . . . . . . . . . 10

I.3.3 Systems . . . . . . . . . . . . . . . . . . . . . . . . 11

I.3.4 Differential calculus; an inverse function theorem . . . . . . . . . . 12

I.4 Dynamic equivalence . . . . . . . . . . . . . . . . . . . . . 15

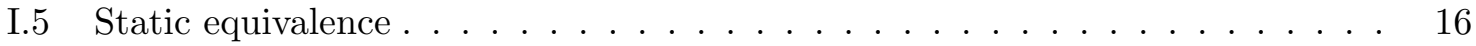

I.6 The single-input case . . . . . . . . . . . . . . . . . . . . . . . . . . 17

I.7 Dynamic linearization . . . . . . . . . . . . . . . . . . . . . . . . . . . . . 19

II Infinitesimal Brunovský form for nonlinear systems with applications to $\begin{array}{ll}\text { dynamic linearization } & 21\end{array}$

II.1 Introduction and Problem Statement . . . . . . . . . . . . . . . . . . . . . 21

II.2 Summary of Chapter I . . . . . . . . . . . . . . . . . . . . . . . . 22

II.3 The Infinitesimal Brunovský Form ． . . . . . . . . . . . . . . . . . . 23

II.4 Dynamic linearization as an integrability problem . . . . . . . . . . . . . . 29

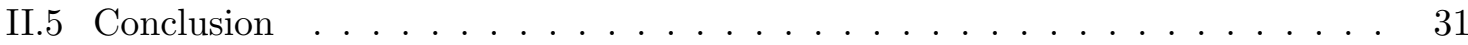




\title{
Chapter I
}

\section{A differential geometric setting for dynamic equivalence and dynamic linearization}

\author{
Jean-Baptiste Pomet
}

\begin{abstract}
This paper presents an (infinite dimensional) geometric framework for control system, based on infinite jet bundles, where a system is represented by a single vector field and dynamic equivalence (to be precise : equivalence by endogenous dynamic feedback) is conjugation by diffeomorphisms. These diffeomorphisms are very much related to Lie-Bäcklund transformations.

It is proved in this framework that dynamic equivalence of single-input systems is the same as static equivalence.
\end{abstract}

Keywords : Dynamic feedback equivalence, dynamic feedback linearization, infinite jet bundles, contact transformations, Lie-Bäcklund transformations, flat systems.

\section{I.1 Introduction}

For a control system

$$
\dot{x}=f(x, u)
$$

where $x \in \mathbb{R}^{n}$ is the state, and $u \in \mathbb{R}^{m}$ is the input, what one usually means by a dynamic feedback is a system with a certain state $z$, input $(x, v)$ and output $u$ :

$$
\begin{aligned}
& \dot{z}=g(x, z, v) \\
& u=\gamma(x, z, v) .
\end{aligned} .
$$

When applying this dynamic feedback to system (I.1), one gets a system with state $(x, z)$ and input $v: \dot{x}=f(x, \gamma(x, z, v)), \dot{z}=g(x, z, v)$. This system may be transformed with 
a change of coordinates $X=\phi(x, z)$ in the extended variables to a system $\dot{X}=h(X, v)$. The problem of dynamic feedback linearization is stated in [7] by B. Charlet, J. Lévine and $\mathrm{R}$. Marino as the one of finding $g, \gamma$ and $\phi$ such that $\dot{X}=h(X, v)$ be a linear controllable system. When $z$ is not present, $\gamma$ and $\phi$ define a static feedback transformation in the usual sense. This transformation is said to be invertible if $\phi$ is a diffeomorphism and $\gamma$ is invertible with respect to $v$; these transformations form a group of transformations. On the contrary, when $z$ is present, the simple fact that the general "dynamic feedback transformation" (I.2), defined by $g, \gamma$ and $\phi$ increases the size of the state prevents dynamic feedbacks in this sense from being "invertible".

In [16, 17], M. Fliess, J. Lévine, P. Martin and P. Rouchon introduced a notion of equivalence in a differential algebraic framework where two systems are equivalent by endogenous dynamic feedback if the two corresponding differential fields are algebraic over one another. This is translated in a state-space representation by some (implicit algebraic) relations between the "new" and the "old" state, output and many derivatives of outputs transforming one system into the other and vice-versa. It is proved that equivalence to controllable linear system is equivalent to differential flatness, which is defined as existence of $m$ elements in the field which have the property to be a "linearizing output" or "flat output". In [26, "Point de vue analytique"], P. Martin introduced the notion of endogenous dynamic feedback as a dynamic feedback (I.2) where, roughly speaking, $z$ is a function of $x, u, \dot{u}, \ddot{u} \ldots$ He proved that a system may be obtained from another one by nonsingular endogenous feedback if and only if there exists a transformation of the same kind as in $[16,17]$ but explicit and analytic which transforms one system into the other. This is called equivalence by endogenous dynamic feedback as in the algebraic case. These transformations may either increase or decrease the dimension of the state.

B. Jakubczyk gives in $[23,24]$ a notion of dynamic equivalence in terms of transformations on "trajectories" of the system; different types of transformations are defined there in terms of infinite jets of trajectories. One of them is proved there to be exactly the one studied here. See after Definition I.1 for further comparisons.

In [36], W.F. Shadwick makes (prior to [16, 17, 23, 24]) a link between dynamic feedback linearization and the notion of absolute equivalence defined by E. Cartan for Pfaffian systems. It is not quite clear that this notion of equivalence coincides with equivalence in the sense of $[16,17]$ or $[23,24]$, the formulation is very different.

The contribution of the present paper -besides Theorem I.3 which states that dynamic equivalent single input systems with the same number of states are static equivalent- is to give a geometric meaning to transformations which are exactly these introduced by P. Martin in [26] (endogenous dynamic feedback transformations). Our system is represented by a single vector field on a certain "infinite-dimensional manifold", and our transformations are diffeomorphisms on this manifold. Then the action of these transformations on systems is translated by the usual transformation diffeomorphisms induce on vector fields. There are of course many technical difficulties in defining vector fields, diffeomorphisms or smooth functions in these "infinite-dimensional manifolds". The original motivation was to "geometrize" the constructions made in $[2,33]$; it grew up into the present framework which, we believe, has some interest in itself, the geometric exposition of $[2,33]$ is contained in the paper reprinted in Chapter II.

Note finally that the described transformations are very closely related to infinite order 
contact transformations or Lie-Bäcklund transformations or $\mathcal{C}$-transformations, see [20, 1] and that the geometric context we present here is the one of infinite jet spaces used in [31, 25, 39, 35] for example to describe and study Lie-Bäcklund transformations. These presentations however are far from being unified, for instance smooth functions do not have to depend only on a finite number of variables in [35], and are not explicitly defined in [1]. They also had to be adapted for many reasons in order to get a technically workable framework; for instance, we prove an inverse function theorem which characterizes local diffeomorphisms without having to refer to an inverse mapping which is of the same type. The language of jet spaces and differential systems has been used already in control theory by M. Fliess [12] and by J.-F. Pommaret [34], with a somewhat different purpose.

Some recent work by M. Fliess [13] (see also a complete exposition on this topic in E. Delaleau's [10]) points out that a more natural state-space representation than (I.1) for a nonlinear system involves not only $x$ and $u$, but also an arbitrary number of time-derivatives of $u$; this is referred to as "generalized-state" representation, and we keep this name for the infinite dimensional state-manifold, see section I.3. In $[13,10]$, the "natural" state-space representation is $F\left(x, \dot{x}, u, \dot{u}, \ddot{u}, \ldots, u^{(J)}\right)=0$ rather than (I.1). Here not only do we suppose that $\dot{x}$ is an explicit function of the other variables ("explicit representation" according to $[13,10]$ ) but also that $J=0$ ("classical representation"). Almost everything in this paper may be adapted to the "non-classical" case, i.e. to the case where some time-derivatives of the input would appear in the right-hand side of (I.1); we chose the classical representation for simplicity and because, as far as dynamic equivalence is concerned, a non-classical system is equivalent to a classical one by simply "adding some integrators"; on the contrary, the implicit case is completely out of the scope of this paper, see the end of section I.2.

Very recently the authors of $[16,17]$ have independently proposed a "differential geometric" approach for dynamic equivalence, see $[18,19]$, which is similar in spirit to the present approach, although the technical results do differ. This was brought to the attention of the author too late for a precise comparison between the two approaches.

The paper is organized as follows : section I.2 presents briefly the point of view of jet spaces and contact structure for system (I.1) considered as a differential relation $\dot{x}-f(x, u)=$ 0 (no theoretical material from this section is used elsewhere in the paper). Section I.3 presents in details the differential structure of the "generalized state-space manifold" where coordinates are $x, u, \dot{u}, \ldots$, where we decide to represent a system by a single vector field. Section I.4 defines in this context dynamic equivalence and relates it to notions already introduced in the literature. Section I.5 deals with static equivalence. Section I.6 is devoted to the single-input case, and states the result that dynamic equivalence and static equivalence are then the same. Finally section I.7 is devoted to dynamic linearization, it introduces in a geometric way the "linearizing outputs" defined for for dynamic linearization in $[16,17,26]$.

\section{I.2 Control systems as differential relations}

This section is only meant to relate the approach described subsequently to some better known theories. It does not contain rigorous arguments.

In the spirit of the work of J. Willems [40], or also of M. Fliess [13], one may consider that the control system (I.1) is simply a differential relation on the functions of time $x(t), u(t)$ and that the object of importance is the set of solutions, i.e. of functions $t \mapsto(x(t), u(t))$ 
such that $\frac{d x}{d t}(t)$ is identically equal to $f(x(t), u(t))$. Of course this description does not need precisely a state-space description like (I.1).

The geometric way of describing the solution of this first order relation in the "independent variable" $t$ (time) and the "dependent variables" $x$ and $u$ is to consider, as in $[1,34,25,39,31$, the fibration

$$
\begin{array}{cl}
\mathbb{R} \times \mathbb{R}^{n+m} & \stackrel{\pi}{\rightarrow} \mathbb{R} \\
(t, x, u) & \mapsto t
\end{array}
$$

and its first jet manifold $J^{1}(\pi)$, which is simply $T\left(\mathbb{R}^{n} \times \mathbb{R}^{m}\right) \times \mathbb{R}$. A canonical set of coordinates on $J^{1}(\pi)$ is $(t, x, u, \dot{x}, \dot{u})$. The relation $R(t, x, u, \dot{x}, \dot{u})=\dot{x}-f(x, u)=0$ defines a sub-manifold $\mathcal{R}$ of the fiber bundle (I.3), which is obviously a sub-bundle. The contact module on $J^{1}(\pi)$ is the module of 1 -forms (or the codistribution) generated by the 1 -forms $\mathrm{d} x_{i}-\dot{x}_{i} \mathrm{~d} t$ and $\mathrm{d} u_{j}-\dot{u}_{j} \mathrm{~d} t, 1 \leq i \leq n, 1 \leq j \leq m$. A "solution" of the differential system is a section $t \mapsto(t, x(t), u(t), \dot{x}(t), \dot{u}(t))$ of the sub-bundle $\mathcal{R}$, which annihilates the contact forms (this simply means that $\frac{\mathrm{d} x}{\mathrm{~d} t}=\dot{x}$ and $\frac{\mathrm{d} u}{\mathrm{~d} t}=\dot{u}$, i.e. that this section is the jet of a section of (I.3)).

Since we wish to consider some transformations involving an arbitrary number of derivatives, we need the infinite jet space $J^{\infty}(\pi)$ of the fibration (I.3). For short, it is the projective limit of the finite jet spaces $J^{k}(\pi)$, and some natural coordinates on this "infinite-dimensional manifold" are $\left(t, x, u, \dot{x}, \dot{u}, \ddot{x}, \ddot{u}, x^{(3)}, u^{(3)}, \ldots \ldots\right)$. The contact forms are

$$
\mathrm{d} x_{i}^{(j)}-x_{i}^{(j+1)} \mathrm{d} t, \quad \mathrm{~d} u_{k}^{(j)}-u_{k}^{(j+1)} \mathrm{d} t \quad\left\{\begin{array}{l}
i=1 \ldots n \\
k=1, \ldots m \\
j \geq 0
\end{array}\right.
$$

This infinite dimensional "manifold" is described in [25] for example, and we will recall in next section what we really need. The "Cartan distribution" is the one annihilated by all these forms, it is spanned by the single vector field

$$
\frac{\partial}{\partial t}+\dot{x} \frac{\partial}{\partial x}+\dot{u} \frac{\partial}{\partial u}+\ddot{x} \frac{\partial}{\partial \dot{x}}+\ddot{u} \frac{\partial}{\partial \dot{u}}+\ldots \ldots
$$

where $\dot{x} \frac{\partial}{\partial x}$ stands for $\sum_{i} \dot{x}_{i} \frac{\partial}{\partial x_{i}}, \dot{u} \frac{\partial}{\partial u}$ for $\sum_{i} \dot{u}_{i} \frac{\partial}{\partial u_{i}} \ldots$ The relation $R$ has to be replaced by its infinite prolongation, i.e. $R$ itself plus all its "Lie derivatives" along (I.5) :

$$
\begin{aligned}
R(t, x, u, \dot{x}, \dot{u}) & =\dot{x}-f(x, u)=0 \\
R_{1}(t, x, u, \dot{x}, \dot{u}, \ddot{x}, \ddot{u}) & =\ddot{x}-\frac{\partial f}{\partial x} \dot{x}-\frac{\partial f}{\partial u} \dot{u}=0 \\
R_{2}\left(t, x, u, \dot{x}, \dot{u}, \ddot{x}, \ddot{u}, x^{(3)}, u^{(3)}\right) & =x^{(3)}-\ldots=0
\end{aligned}
$$

This defines a sub-bundle $\mathcal{R}_{\infty}$ of $J^{\infty}(\pi)$. A "solution" of the differential system is a section $t \mapsto(t, x(t), u(t), \dot{x}(t), \dot{u}(t), \ddot{x}(t), \ddot{u}(t), \ldots)$ of the sub-bundle $\mathcal{R}_{\infty}$, which annihilates the contact forms; it is obviously defined uniquely by $x(t)$ and $u(t)$ such that $\frac{d x}{d t}(t)=f(x(t), u(t))$ with the functions $u^{(j)}$ and $x^{(j)}$ obtained by differentiating $x(t)$ and $u(t)$.

$\mathcal{R}_{\infty}$ is a sub-bundle of $J^{\infty}(\pi)$ which has a particular form : since the relations allow one to explicitly express all the time-derivatives $\dot{x}, \ddot{x}, x^{(3)}, \ldots$ of $x$ as functions of $x, u, \dot{u}, \ddot{u}, u^{(3)}, \ldots$, a natural set of coordinates on this sub-manifold is $(t, x, u, \dot{u}, \ddot{u}, \ldots)$; note that if, instead of 
the explicit form (I.1), we had an implicit system $f(x, u, \dot{u})=0$, this would not be true. The vector field (I.5), which spans the Cartan distribution is tangent to $\mathcal{R}_{\infty}$, and its expression in the coordinates $(t, x, u, \dot{u}, \ddot{u}, \ldots)$ considered as coordinates on $\mathcal{R}_{\infty}$ is

$$
\frac{\partial}{\partial t}+f(x, u) \frac{\partial}{\partial x}+\dot{u} \frac{\partial}{\partial u}+\ddot{u} \frac{\partial}{\partial \dot{u}}+\ldots+u^{(k+1)} \frac{\partial}{\partial u^{(k)}}+\ldots \ldots
$$

and the restriction of the contact forms are $\mathrm{d} x-f \mathrm{~d} t, \mathrm{~d} u^{(j)}-u^{j+1} \mathrm{~d} t, j \geq 0$. The sub-bundles $\mathcal{R}_{\infty}$ obtained for different systems are therefore all diffeomorphic to a certain "canonical object" independent of the system, and where coordinates are $(t, x, u, \dot{u}, \ddot{u}, \ldots)$, let this object be $\mathbb{R} \times \mathcal{M}_{\infty}^{m, n}$ where $\mathcal{M}_{\infty}^{m, n}$ is described in more details in next section and the first factor $\mathbb{R}$ is time, with an embedding $\psi$ of $\mathbb{R} \times \mathcal{M}_{\infty}^{m, n}$ into $J_{\infty}(\pi)$ which defines a diffeomorphism between $\mathcal{R}_{\infty}$ and $\mathbb{R} \times \mathcal{M}_{\infty}^{m, n}$; this embedding depends on the system and completely determines it; it pulls back the contact module on $J^{\infty}(\pi)$ to a certain module of forms on $\mathbb{R} \times \mathcal{M}_{\infty}^{m, n}$ and the Cartan vector field (I.5) into (I.7). The points in $J^{\infty}(\pi)$ which are outside $\mathcal{R}_{\infty}$ are not really of interest to the system, so that we only need to retain $\mathcal{R}_{\infty}$, and it turns out that all the information is contained in $\mathbb{R} \times \mathcal{M}_{\infty}^{m, n}$ and the vector field (I.7) which translates the way the contact module is pulled back by the embedding of $\mathbb{R} \times \mathcal{M}_{\infty}^{m, n}$ into $J_{\infty}(\pi)$ whose image is $\mathcal{R}_{\infty}$. This is the point of view defended in [39] for example where such a manifold endowed with what it inherits from the contact structure on $J^{\infty}(\pi)$ is called a "diffiety". It is only in the special case of explicit systems like (I.1) that all diffieties can be parameterized by $x, u, \dot{u}, \ldots$ and therefore can all be represented by the single object $\mathcal{M}_{\infty}^{m, n}$, endowed with a contact structure, or a Cartan vector field, which of course depends on the system.

Finally, since everything is time-invariant, one may "drop" the variable $t$ (or quotient by time-translations, or project on the sub-manifold $\{t=0\}$ which is possible because all objects are invariant along the fibers) and work with the coordinates $(x, u, \dot{u}, \ddot{u}, \ldots)$ only, with $f \frac{\partial}{\partial x}+\dot{u} \frac{\partial}{\partial u}+\ddot{u} \frac{\partial}{\partial \dot{u}}+\ldots$ instead of (I.7); solutions are curves which are tangent to this vector field. This is the point of view we adopt here, and this is described in details in next section.

\section{I.3 The generalized state-space manifold}

The phrase "generalized state" denotes the use of many derivatives of the input as in $[13,10]$. The "infinite-dimensional manifold" $\mathcal{M}_{\infty}^{m, n}$ we are going to consider is parameterized by $x, u, \dot{u}, \ddot{u}, \ldots$; in order to keep things simple, we define it in coordinates, i.e. a point of $\mathcal{M}_{\infty}^{m, n}$ is simply a sequence of numbers, as in [30] for example. It may be extended to $x$ and $u$ living in arbitrary manifolds via local coordinates, but, since dynamic equivalence is local in nature, the present description is suitable.

\section{I.3.1 The manifold, functions and mappings}

For $k \geq-1$, let $\mathcal{M}_{k}^{m, n}$ be $\mathbb{R}^{n} \times\left(\mathbb{R}^{m}\right)^{k+1}\left(\mathcal{M}_{-1}^{m, n}\right.$ is $\left.\mathbb{R}^{n}\right)$, and let us denote the coordinates in $\mathcal{M}_{k}^{m, n}$ by

$$
\left(x, u, \dot{u}, \ddot{u}, \ldots, u^{(k)}\right)
$$

where $x$ is in $\mathbb{R}^{n}$ and $u, \dot{u}, \ldots$ are in $\mathbb{R}^{m} . \mathcal{M}_{\infty}^{m, n}$ is the space of infinite sequences

$$
\left(x, u, \dot{u}, \ddot{u}, \ldots, u^{(j)}, u^{(j+1)}, \ldots\right) \text {. }
$$


For simplicity, we shall use the following notation :

$$
\mathcal{U}=\left(u, \dot{u}, \ddot{u}, u^{(3)}, \ldots\right), \quad \mathcal{X}=(x, \mathcal{U})=\left(x, u, \dot{u}, \ddot{u}, u^{(3)}, \ldots\right) .
$$

Let, for $k \geq-1$, the projection $\pi_{k}$, from $\mathcal{M}_{\infty}^{m, n}$ to $\mathcal{M}_{k}^{m, n}$ be defined by :

$$
\begin{aligned}
\pi_{k}(\mathcal{X}) & =\left(x, u, \dot{u}, \ldots, u^{(k)}\right) \quad k \geq 0 \\
\pi_{-1}(\mathcal{X}) & =x .
\end{aligned}
$$

$\mathcal{M}_{\infty}^{m, n}$ may be constructed as the projective limit of $\mathcal{M}_{k}^{m, n}$, and this naturally endows it with the weakest such that all these projections are continuous (product topology); a basis of the topology are the sets

$$
\pi_{k}^{-1}(O), \quad O \text { open subset of } \mathcal{M}_{k}^{m, n} \text {. }
$$

This topology makes $\mathcal{M}_{\infty}^{m, n}$ a topological vector space, which is actually a Fréchet space (see for instance [4]). It is easy to see that continuous linear forms are these which depend only on a finite number of coordinates. This leads one to the (false) idea that there is a natural way of defining differentiability so that differentiable functions depend only on a finite number of variables, which is exactly the class of smooth functions we wish to consider (as in most of the literature on differential system and jet spaces $[1,25,30,31,39]$ ), since they translate into realistic dynamic feedbacks from the system theoretic point of view. It is actually possible to define a very natural notion of differentiability in Fréchet spaces (see for instance the very complete [21]) but there is nothing wrong in this framework with smooth functions depending on infinitely many variables. For instance the function mapping $\left(u, \dot{u}, \ddot{u}, u^{(3)}, \ldots\right)$ to $\sum_{j=0}^{\infty} \frac{1}{2^{j}} \rho\left(\frac{u^{(j)}}{j}\right)$, with $\rho$ a smooth function with compact support containing 0 vanishing at 0 as well as its derivatives of all orders depends on all the variables at zero, but it is smooth in this framework. It is hard to imagine a local definition of differentiability which would classify this function non-smooth.

Here, we do not wish to consider smooth functions or smooth maps depending on infinitely many variables; we therefore define another differentiable structure, which agrees with the one usually used for differential systems $[31,1,30,25,39]$ :

- A function $h$ from an open subset $V$ of $\mathcal{M}_{\infty}^{m, n}$ to $\mathbb{R}$ (or to any finite-dimensional manifold) is a smooth function at $\mathcal{X} \in V$ if and only if, locally at each point, it depends only on a finite number of derivatives of $u$ and, as a function of a finite number of variables, it is smooth (of class $\mathcal{C}^{\infty}$ ); more technically : if and only if there exists an open neighborhood $U$ of $\mathcal{X}$ in $V$, an integer $\rho$, and a smooth function $h_{\rho}$ from an open subset of $\mathcal{M}_{\rho}^{m, n}$ to $\mathbb{R}$ (or to the finite-dimensional manifold under consideration) such that $h(\mathcal{Y})=h_{\rho} \circ \pi_{\rho}(\mathcal{Y})$ for all $\mathcal{Y}$ in $U$. It is a smooth function on $V$ if it is a smooth function at all $\mathcal{X}$ in $V$. The highest $\rho$ such that $h$ actually depends on the $\rho$ th derivative of $u$ on any neighborhood of $\mathcal{X}(-1$ if it depends on $x$ only on a certain neighborhood of $\mathcal{X}$ ) we will call the order of $h$ at $\mathcal{X}$, and we denote it by $\delta(h)(\mathcal{X})$. It is also the largest integer such that $\frac{\partial h}{\partial u^{(\rho)}}$ (this may be defined in coordinates and is obviously a smooth function) is not identically zero on any neighborhood of $\mathcal{X}$. Note that $\delta(h)$ may be unbounded on $\mathcal{M}_{\infty}^{m, n}$. We denote by $\mathcal{C}^{\infty}(V)$ the algebra of smooth functions from $V$ to $\mathbb{R}, \mathcal{C}^{\infty}\left(\mathcal{M}_{\infty}^{m, n}\right)$ if $V=\mathcal{M}_{\infty}^{m, n}$. 
- A smooth mapping from an open subset $V$ of $\mathcal{M}_{\infty}^{m, n}$ to $\mathcal{M}_{\infty}^{\tilde{m}, \tilde{n}}$ is a map $\varphi$ from $V$ to $\mathcal{M}_{\infty}^{\tilde{m}, \tilde{n}}$ such that, for any $\psi$ in $\mathcal{C}^{\infty}\left(\mathcal{M}_{\infty}^{\widetilde{m}, \tilde{n}}\right), \psi \circ \varphi$ is in $\mathcal{C}^{\infty}(V)$. It is a smooth mapping at $\mathcal{X}$ if it is a smooth mapping from a certain neighborhood of $\mathcal{X}$ to $\mathcal{M}_{\infty}^{\tilde{m}, \tilde{n}}$. Of course, in coordinates, it is enough that this be true for $\psi$ any coordinate function. For such a map and for all $k$, there exists locally an integer $\rho_{k}$ and a (unique) smooth map $\varphi_{k}$ from $\pi_{\rho_{k}}(V) \subset \mathcal{M}_{\rho_{k}}^{m, n}$ to $\mathcal{M}_{k}^{\tilde{m}, \widetilde{n}}$ such that

$$
\pi_{k} \circ \varphi=\varphi_{k} \circ \pi_{\rho_{k}} .
$$

The smallest possible $\rho_{k}$ at a point $\mathcal{X}$ is $\delta\left(\pi_{k} \circ \varphi\right)(\mathcal{X})$.

- A diffeomorphism from an open subset $V$ of $\mathcal{M}_{\infty}^{m, n}$ to an open subset $\widetilde{V}$ of $\mathcal{M}_{\infty}^{\tilde{m}, \tilde{n}}$ is a smooth mapping $\varphi$ from $V$ to $\widetilde{V}$ which is invertible and is such that $\varphi^{-1}$ is a smooth mapping from $\widetilde{V}$ to $V$.

- A static diffeomorphism $\varphi$ from an open subset $V$ of $\mathcal{M}_{\infty}^{m, n}$ to an open subset $\tilde{V}$ of $\mathcal{M}_{\infty}^{\widetilde{m}, \tilde{n}}$ is a diffeomorphism from $V$ to $\widetilde{V}$ such that for all $k, \delta\left(\pi_{k} \circ \varphi\right)(\mathcal{X})$ is constant equal to $k$.

- A (local) system of coordinates on $\mathcal{M}_{\infty}^{m, n}$ (at a certain point) is a sequence $\left(h_{\alpha}\right)_{\alpha \geq 0}$ of smooth functions (defined on a neighborhood of the point under consideration) such that the smooth mapping $\mathcal{X} \mapsto\left(h_{\alpha}(\mathcal{X})\right)_{\alpha \geq 0}$ is a local diffeomorphism onto an open subset of $\mathbb{R}^{\mathbb{N}}$, considered as $\mathcal{M}_{\infty}^{1,0}$.

Note that the functions $x_{1}, \ldots, x_{n}, u_{1}, \ldots, u_{m}, \dot{u}_{1}, \ldots, \dot{u}_{m}, \ldots$ are coordinates in this sense. Actually, this makes all the "manifolds" $\mathcal{M}_{\infty}^{m, n}$ globally diffeomorphic to $\mathcal{M}_{\infty}^{1,0}$, so that they are all diffeomorphic to one another (this can be viewed as renumbering the natural coordinates). The following proposition shows that static diffeomorphisms are much more restrictive: they preserve $n$ and $m$.

Proposition I.1 Let $\varphi$ be a static diffeomorphism from an open set $U$ of $\mathcal{M}_{\infty}^{m, n}$ to an open set $V$ of $\mathcal{M}_{\infty}^{\widetilde{m}, \tilde{n}}$. Its inverse $\varphi^{-1}$ is also a static diffeomorphism and $\varphi$ induces, for all $k \geq 0$, a diffeomorphism $\varphi_{k}$ from $\mathcal{M}_{k}^{m, n}$ to $\mathcal{M}_{k}^{\tilde{m}, \tilde{n}}$ (from $\mathbb{R}^{n}$ to $\mathbb{R}^{\tilde{n}}$ for $k=-1$ ). Its existence therefore implies $\widetilde{n}=n$ and $\widetilde{m}=m$.

Proof : For all $k \geq-1$, since $\delta\left(\varphi \circ \pi_{k}\right)=k$, there exists a mapping $\varphi_{k}$ from $\pi_{k}(U)$ to $\pi_{k}(V)$ satisfying (I.10) with $\rho_{k}=k$. All these mappings are onto because if one of them was not onto, (I.10) would imply that $\varphi$ is onto either. Now let us consider $\varphi^{-1}$; it is a diffeomorphism from $V$ to $U$ and there exists therefore, for all $k$, an integer $\sigma_{k}$ and a smooth map $\left(\varphi^{-1}\right)_{k}$ from $\pi_{\sigma_{k}}(V) \subset \mathcal{M}_{\sigma_{k}}^{\tilde{m}, \tilde{n}}$ to $\mathcal{M}_{k}^{m, n}$ such that

$$
\pi_{k} \circ \varphi^{-1}=\left(\varphi^{-1}\right)_{k} \circ \pi_{\sigma_{k}} .
$$

Applying $\varphi$ on the right to both sides and using the fact that $\pi_{\sigma_{k}} \circ \varphi=\varphi_{\sigma_{k}} \circ \pi_{\sigma_{k}}$, we get

$$
\pi_{k}=\left(\varphi^{-1}\right)_{k} \circ \varphi_{\sigma_{k}} \circ \pi_{\sigma_{k}}
$$


Applied to $(x, u, \dot{u}, \ldots)$, this means

$$
\begin{aligned}
\left(x, u, \dot{u}, \ldots, u^{(k)}\right) & =\left(\varphi^{-1}\right)_{k}\left(y, v, \dot{v}, \ldots, v^{(k)}, \ldots, v^{\left(\sigma_{k}\right)}\right) \\
\text { with }\left(y, v, \dot{v}, \ldots, v^{(k)}, \ldots, v^{\left(\sigma_{k}\right)}\right) & =\varphi_{\sigma_{k}}\left(x, u, \dot{u}, \ldots, u^{(k)}, \ldots, u^{\left(\sigma_{k}\right)}\right)
\end{aligned}
$$

Since $\varphi_{\sigma_{k}}$ is onto and each $v^{(j)}$ depends only on $x, u, \ldots, u^{(j)},\left(\right.$ I.13) implies that $\left(\varphi^{-1}\right)_{k}$ depends only on $y, v, \dot{v}, \ldots, v^{(k)}$. Therefore $\sigma_{k}$ might have been taken to be $k$, and then one has (I.12) with $\sigma_{k}=k$ and therefore

$$
\left(\varphi^{-1}\right)_{k} \circ \varphi_{k}=\operatorname{Id} \underset{\mathcal{M}_{k}^{\tilde{m}, \tilde{m}}}{ }
$$

which proves that each $\varphi_{k}$ is a diffeomorphism and ends the proof.

Let us define, as examples of diffeomorphisms, the (non static!) diffeomorphisms $\Upsilon_{n,\left(p_{1}, \ldots, p_{m}\right)}$ from $\mathcal{M}_{\infty}^{m, n} \mathcal{M}_{\infty}^{m, n+p_{1}+\ldots+p_{m}}$ which "adds $p_{k}$ integrators on the $k$ th input" :

$$
\Upsilon_{n,\left(p_{1}, \ldots, p_{m}\right)}(x, \mathcal{U})=(z, \mathcal{V}) \text { with } \begin{aligned}
& z=\left(x, u_{1}, \dot{u}_{1} \ldots u_{1}^{\left(p_{1}-1\right)}, \ldots, u_{m}, \dot{u}_{m}, \ldots u_{m}^{\left(p_{m}-1\right)}\right) \\
& v_{k}^{(j)}=u_{k}^{\left(j+p_{k}\right)}
\end{aligned}
$$

It is invertible : one may define $\Upsilon_{N,\left(-p_{1}, \ldots,-p_{m}\right)}$ from $\mathcal{M}_{\infty}^{m, N}$ to $\mathcal{M}_{\infty}^{m, N-p_{1}-\ldots-p_{m}}$ for $N \geq$

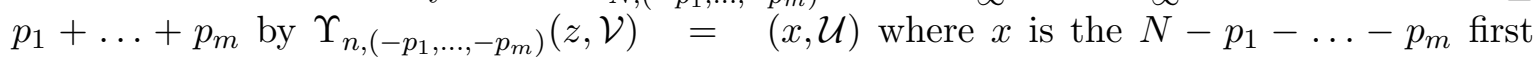
coordinates of $z$, and $u_{k}^{(j)}$ is $v_{k}^{\left(j-p_{k}\right)}$ if $j \geq p_{k}$ and one of the remaining components of $z$ if $0 \leq j \leq p_{k}-1$, so that $\Upsilon_{n,\left(p_{1}, \ldots, p_{m}\right)} \circ \Upsilon_{n,\left(-p_{1}, \ldots,-p_{m}\right)}=\mathrm{Id}$.

\section{I.3.2 Vector fields and differential forms}

The "tangent bundle" to the infinite dimensional manifold $\mathcal{M}_{\infty}^{m, n}$ is, since $\mathcal{M}_{\infty}^{m, n}$ is a vector space, $\mathcal{M}_{\infty}^{m, n} \times \mathcal{M}_{\infty}^{m, n}$, which is a (trivial) vector bundle over $\mathcal{M}_{\infty}^{m, n}$. A smooth vector field is a smooth (as a mapping from $\mathcal{M}_{\infty}^{m, n}$ to $\mathcal{M}_{\infty}^{m, n} \times \mathcal{M}_{\infty}^{m, n}$, considered as $\mathcal{M}_{\infty}^{2 m, 2 n}$ ) section of this bundle. It is of the form

$$
F=f \frac{\partial}{\partial x}+\sum_{0}^{\infty} \alpha_{j} \frac{\partial}{\partial u^{(j)}}
$$

where $f$ is a smooth function from $\mathcal{M}_{\infty}^{m, n}$ to $\mathbb{R}^{n}$ and the $\alpha_{j}$ 's are smooth functions from $\mathcal{M}_{\infty}^{m, n}$ to $\mathbb{R}^{m}$, where $f \frac{\partial}{\partial x}$ stands for $\sum_{i} f_{i} \frac{\partial}{\partial x_{i}}$ and $\alpha_{j} \frac{\partial}{\partial u^{(j)}}$ for $\sum_{i} \alpha_{j, i} \frac{\partial}{\partial u_{i}^{(j)}}$, and the $\frac{\partial}{\partial x_{i}}$ 's and $\frac{\partial}{\partial u_{i}^{(j)}}$ 's are the canonical sections corresponding to the "coordinate vector fields" associated with the canonical coordinates. Vector fields obviously define smooth differential operators on smooth functions : in coordinates, $L_{F} h$ is an infinite sum with finitely many nonzero terms.

Smooth differential forms are smooth sections of the cotangent bundle, which is simply $\mathcal{M}_{\infty}^{m, n} \times\left(\mathcal{M}_{\infty}^{m, n}\right)^{*}$ where $\left(\mathcal{M}_{\infty}^{m, n}\right)^{*}$ is the topological dual of $\mathcal{M}_{\infty}^{m, n}$, i.e. the space of infinite sequences with only a finite number of nonzero entries; they can be written :

$$
\omega=g \mathrm{~d} x+\sum_{\text {finite }} \beta_{j} \mathrm{~d} u^{(j)}
$$


This defines the $\mathcal{C}^{\infty}\left(\mathcal{M}_{\infty}^{m, n}\right)$ module $\Lambda^{1}\left(\mathcal{M}_{\infty}^{m, n}\right)$ of smooth differential forms on $\mathcal{M}_{\infty}^{m, n}$. One may also define differential forms of all degree.

Of course, one may apply a differential form to a vector field according to $\langle\omega, F\rangle=$ $f g+\sum \alpha_{j} \beta_{j}$ (compare (I.16)-(I.17)), where the sum is finite because finitely many $\beta_{j}$ 's are nonzero. One may also define the Lie derivative of a smooth function $h$, of a differential form $\omega, \ldots$ along a vector field $F$, which we denote by $L_{F} h$ or $L_{F} \omega$. The Lie bracket of two vector fields may also be defined. All this may be defined exactly as in the finite-dimensional case because, on a computational point of view, all the sums to be computed are finite.

Finally, note that a diffeomorphism carries differential forms, vector fields, functions from a manifold to another, exactly as in the finite dimensional case; for example, if $\varphi$ is a diffeomorphism from $\mathcal{M}_{\infty}^{m, n}$ to $\mathcal{M}_{\infty}^{\widetilde{m}, \tilde{n}}, F$ is given by (I.16) and $z, v, \dot{v}, \ddot{v}, \ldots$ are the canonical coordinates on $\mathcal{M}_{\infty}^{\widetilde{m}, \tilde{n}}$, the vector field $\varphi_{*} F$ on $\mathcal{M}_{\infty}^{\tilde{m}, \widetilde{n}}$ is given by $\sum_{i} \widetilde{f}_{i} \frac{\partial}{\partial x_{i}}+\sum_{j, k} \widetilde{\alpha}_{j, k} \frac{\partial}{\partial u_{k}^{(j)}}$ with $\widetilde{f}_{i}=\left(L_{F}\left(z_{i} \circ \varphi\right)\right) \circ \varphi^{-1}$ and $\widetilde{\alpha}_{j, k}=\left(L_{F}\left(v_{k}^{(j)} \circ \varphi\right)\right) \circ \varphi^{-1}$.

\section{I.3.3 Systems}

A system is a vector field $F$ on $\mathcal{M}_{\infty}^{m, n}$-with $n \geq 0$ and $m \geq 1$ some integers- of the form

$$
F(\mathcal{X})=f(x, u) \frac{\partial}{\partial x}+\sum_{j=0}^{+\infty} u^{(j+1)} \frac{\partial}{\partial u^{(j)}}
$$

i.e. the $x$-component of $F$ is a function of $x$ and $u$ only, and its $u^{(j)}$-component is $u^{(j+1)}$. This may be rewritten, in a more condensed form,

$$
F=f+C
$$

where $C$ is the canonical vector field on $\mathcal{M}_{\infty}^{m, n}$, given by

$$
C=\sum_{0}^{\infty} u^{(j+1)} \frac{\partial}{\partial u^{(j)}}
$$

and the vector field $f$ is such that

$$
\begin{gathered}
\left\langle\mathrm{d} u_{i}^{(j)}, f\right\rangle=0 \quad i=1, \ldots, m, j \geq 0 \\
{\left[\frac{\partial}{\partial u_{i}^{(j)}}, f\right]=0 \quad i=1, \ldots, m, j \geq 1 .}
\end{gathered} .
$$

$m$ will be called the number of inputs of the system, and $n$ its state dimension. Note that in the (explicit) non-classical case $[13,10]$ (i.e. the case when some derivatives of $u$ would appear in the right-hand side of (I.1), there would be no restriction on $f$, besides being smooth, i.e. the second relation in (I.21) would no longer be there (note however that any smooth vector field has zero Lie Bracket with $\frac{\partial}{\partial u^{(j)}}$ for $j$ large enough, or in other words $f$ depending on infinitely many time-derivatives of $u$ in (I.1) is ruled out).

In the special case where $n=0$, there is only one system (with "no state") on $\mathcal{M}_{\infty}^{m, 0}$. We call this system the canonical linear system with $m$ inputs; it is simply represented by the canonical vector field $C$ given by (I.20). 
In section I.2, a system was an embedding of $\mathbb{R} \times \mathcal{M}_{\infty}^{m, n}$ as a sub-bundle of $J^{\infty}$; this defines canonically the vector field $F$ on $\mathcal{M}_{\infty}^{m, n}$ as, more or less, the pull back of the Cartan vector field (annihilating the contact forms) in $J^{\infty}(\pi)$.

$F$ is the vector field defining the "total derivation along the system", i.e. the derivative of a smooth function (depending on $x, u, \dot{u}, \ldots, u^{(j)}$ ) knowing that $\dot{x}=f(x, u)$ is exactly its Lie derivative along this vector field. In [23], B. Jakubczyk attaches a differential algebra to the smooth system (I.1) which is exactly $\mathcal{C}^{\infty}\left(\mathcal{M}_{\infty}^{m, n}\right)$ endowed with the Lie derivative along the vector field $F$. Of course, this is very much related to the differential algebraic approach introduced in control theory by M. Fliess [13], based on differential Galois theory, and where a system is represented by a certain differential field. In the analytic case, as explained in [8], this differential field may be realized as the field of fractions of the integral domain $\mathcal{C}^{\omega}\left(\mathcal{M}_{\infty}^{m, n}\right)$. The present framework is more or less dual to these differential algebra representations since it describes the set of "points" on which the objects manipulated in differential algebra are "functions".

The following proposition gives an intrinsic definition of the number of inputs, which will be useful to prove that it is invariant under dynamic equivalence :

Proposition I.2 The number of inputs $m$ is the largest integer $q$ such that there exists $q$ smooth functions $h_{1}, \ldots, h_{q}$ from $\mathcal{M}_{\infty}^{m, n}$ to $\mathbb{R}$ such that all the functions

$$
L_{F}^{j} h_{k} \quad 1 \leq k \leq q, j \geq 0
$$

are independent (the Jacobian of a finite collection of them has maximum rank).

Proof : On one hand, $h_{k}(x, \mathcal{U})=u_{k}$ provides $m$ functions enjoying this property. On the other hand, consider $m+1$ smooth functions $h_{1}, \ldots, h_{m+1}$, let $\rho \geq 0$ be such that they are functions only of $x, u, \dot{u}, \ldots, u^{(\rho)}$, and consider the $(m+1)(n+m \rho+1)$ functions functions

$$
L_{F}^{j} h_{k} \quad 1 \leq k \leq m+1,0 \leq j \leq n+m \rho ;
$$

from the form of $F$ (see (I.19) and (I.20)), they depend only on $x, u, \dot{u}, \ldots, u^{(\rho+n+m \rho)}$, i.e. on $n+m(\rho+n+m \rho+1)$ coordinates; since this integer is strictly smaller than $(m+1)(n+m \rho+1)$, the considered functions cannot be independent.

\section{I.3.4 Differential calculus; an inverse function theorem}

All the identities from differential calculus involving functions, vector fields, differential forms apply on the "infinite-dimensional manifold" $\mathcal{M}_{\infty}^{m, n}$ exactly as if it were finite-dimensional : if it is an equality between functions or forms, it involves only a finite number of variables (i.e. both sides are constant along the vector fields $\frac{\partial}{\partial u_{k}^{(j)}}$ for $j$ larger than a certain $J>0$ ) so that all the vector fields appearing in the formula may be truncated (replaced by a vector fields with a zero component on $\frac{\partial}{\partial u_{k}^{(j)}}$ for $\left.j>J\right)$, and everything may then be projected by a certain $\pi_{K}$ ( $K$ possibly larger than $J$ ), yielding an equivalent formula on the finitedimensional manifold $\mathcal{M}_{K}^{m, n}$; if it is an equality between vector fields, it may be checked component by component, yielding equalities between functions, and the preceding remark applies. 
Of course, theorems from differential calculus yielding existence of an object do not follow so easily, and often do not hold in infinite dimension. For instance, locally around a point where it is nonzero, a vector field on a manifold of dimension $n$ has $n-1$ independent first integrals (functions whose Lie derivative along this vector field is zero) whereas this is false on $\mathcal{M}_{\infty}^{m, n}$ in general: for the vector field $C$ on $\mathcal{M}_{\infty}^{m, 0}$ given by (I.20), any function $h$ such that $L_{C} h=0$ is a constant function.

One fundamental theorem in differential calculus is the inverse function theorem stating that a smooth function from a manifold to another one whose tangent map at a certain point is an isomorphism admits locally a smooth inverse. In infinite dimension, the situation is to more intricate, see for instance [21] for a very complete discussion of this subject and general inverse function theorems on Fréchet spaces, which are not exactly the kind of theorem we will need since more general smooth functions are considered there. Here, for a mapping $\varphi$ from $\mathcal{M}_{\infty}^{m, n}$ (coordinates: $\left.x, u, \dot{u}, \ldots\right)$ to $\mathcal{M}_{\infty}^{\widetilde{m}, \widetilde{n}}$ (coordinates: $z, v, \dot{v}, \ldots$ ), the function assigning to each point the tangent map to $F$ at this point may be represented by the collection of differential forms $\mathrm{d}\left(z_{i} \circ \varphi\right), \mathrm{d}\left(v_{k}^{(j)} \circ \varphi\right)$, and a way of saying that, at all point, the linear mapping is invertible with a continuous inverse, and that it depends smoothly on the point, is to say that these forms are a basis of the module $\Lambda^{1}\left(\mathcal{M}_{\infty}^{m, n}\right)$; equivalently, this tangent map might be represented by an infinite matrix whose lines are finite (each line represents one of the above differential forms), and which is invertible for matrix multiplication with an inverse having also finite lines. It is clear that for a diffeomorphism this linear invertibility holds; the additional assumption we add to get a converse is that the mapping under consideration carries a control system (as defined by (I.19)) on $\mathcal{M}_{\infty}^{m, n}$ to a control system on $\mathcal{M}_{\infty}^{\widetilde{m}, \tilde{n}}$; note also that we require that the tangent map be invertible in a neighborhood of the point under consideration whereas the finite-dimensional theorem just asks for invertibility at the point.

Besides its intrinsic interest, the following result will be required to prove theorem I.5 which characterizes "linearizing outputs" in terms of their differentials.

Proposition I.3 (local inverse function Theorem) Let $m, n, \widetilde{m}, \widetilde{n}$ be nonnegative integers with $m$ and $\widetilde{m}$ nonzero. Let $z_{1}, \ldots, z_{\widetilde{n}}, v_{1}, \ldots, v_{\widetilde{m}}, \dot{v}_{1}, \ldots, \dot{v}_{\widetilde{m}}, \ldots$ be the canonical coordinates on $\mathcal{M}_{\infty}^{\tilde{m}, \tilde{n}}$, and $\overline{\mathcal{X}}=(\bar{x}, \bar{u}, \dot{\bar{u}}, \ddot{\bar{u}}, \ldots)$ be a point in $\mathcal{M}_{\infty}^{m, n}$. Let $\varphi$ be a smooth mapping from a neighborhood of $\overline{\mathcal{X}}$ in $\mathcal{M}_{\infty}^{m, n}$ to a neighborhood of $\varphi(\overline{\mathcal{X}})$ in $\mathcal{M}_{\infty}^{\tilde{m}, \tilde{n}}$ such that

1. on a neighborhood of $\overline{\mathcal{X}}$, the following set of 1-forms on $\mathcal{M}_{\infty}^{m, n}$ :

$$
\left\{\mathrm{d}\left(z_{i} \circ \varphi\right)\right\}_{1 \leq i \leq \tilde{n}} \cup\left\{\mathrm{d}\left(v_{k}^{(j)} \circ \varphi\right)\right\}_{1 \leq k \leq \widetilde{m}, j \geq 0},
$$

form a basis of the $\mathcal{C}^{\infty}\left(\mathcal{M}_{\infty}^{m, n}\right)$-module $\Lambda^{1}\left(\mathcal{M}_{\infty}^{m, n}\right)$,

2. there exists two control systems $F$ on $\mathcal{M}_{\infty}^{m, n}$ and $\widetilde{F}$ on $\mathcal{M}_{\infty}^{\tilde{m}, \tilde{n}}$ such that, for all function $\widetilde{h} \in \mathcal{C}^{\infty}\left(\mathcal{M}_{\infty}^{\tilde{m}, \tilde{n}}\right)$, defined on a neighborhood of $\varphi(\overline{\mathcal{X}})$,

$$
\left(L_{\widetilde{F}} \widetilde{h}\right) \circ \varphi=L_{F}(\widetilde{h} \circ \varphi) .
$$

Then $\varphi$ is a local diffeomorphism at $\overline{\mathcal{X}}$, i.e. there exists a neighborhood $U$ of $\overline{\mathcal{X}}$ in $\mathcal{M}_{\infty}^{m, n}$, a neighborhood $V$ of $\varphi(\overline{\mathcal{X}})$ in $\mathcal{M}_{\infty}^{\widetilde{m}, \tilde{n}}$ and a smooth mapping (a diffeomorphism) $\psi$ from $V$ to $U$ such that $\psi \circ \varphi=I d_{U}$ and $\varphi \circ \psi=I d_{V}$. 
Note that (I.22) is a way of expressing that the tangent map to $\varphi$ is invertible with a continuous inverse, and (I.23) is a way of expressing that $\varphi$ transforms the control system $F$ into the control system $\widetilde{F}$, in a dual manner since writing $\widetilde{F}=\varphi_{*} F$ would presuppose that $\varphi$ is a diffeomorphism.

Proof : Let $x_{1}, \ldots, x_{n}, u_{1}, \ldots, u_{m}, \dot{u}_{1}, \ldots, \dot{u}_{m}, \ldots \ldots$ be the canonical coordinates on $\mathcal{M}_{\infty}^{m, n}$. The first condition implies that there exist some smooth functions $a_{i}^{k}, b_{i}^{k, j}, c_{i}^{k}, d_{i}^{k, j}$ such that

$$
\begin{aligned}
& \mathrm{d} x_{i}=\sum_{k=1}^{\widetilde{n}} a_{i}^{k} \mathrm{~d}\left(z_{k} \circ \varphi\right)+\sum_{j=0}^{L} \sum_{i=1}^{\tilde{m}} b_{i}^{k, j} \mathrm{~d}\left(v_{k}^{(j)} \circ \varphi\right) \quad i=1, \ldots, n \\
& \mathrm{~d} u_{i}=\sum_{k=1}^{\widetilde{n}} c_{i}^{k} \mathrm{~d}\left(z_{k} \circ \varphi\right)+\sum_{j=0}^{L} \sum_{i=1}^{\widetilde{m}} d_{i}^{k, j} \mathrm{~d}\left(v_{k}^{(j)} \circ \varphi\right) \quad i=1, \ldots, m .
\end{aligned}
$$

Let $K$ be the integer such that the functions $z_{1} \circ \varphi, \ldots, z_{\widetilde{n}} \circ \varphi, v_{1} \circ \varphi, \ldots, v_{\widetilde{m}} \circ \varphi, \ldots, v_{1}^{(L)} \circ$ $\varphi, \ldots, v_{\widetilde{m}}^{(L)} \circ \varphi$, and the functions $a_{i}^{k}, b_{i}^{k, j}, c_{i}^{k}, d_{i}^{k, j}$ all depend on $x, u, \dot{u}, \ldots, u^{(K)}$ only. Then $z_{1} \circ \varphi, \ldots, z_{\widetilde{n}} \circ \varphi, v_{1} \circ \varphi, \ldots, v_{\widetilde{m}} \circ \varphi$ are $\widetilde{n}+\widetilde{m}$ functions of the $n+(K+1) m$ variables $x_{1}, \ldots, x_{n}, u_{1}, \ldots, u_{m}, \ldots, u_{1}^{(K)}, \ldots, u_{m}^{(K)}$ which, from condition 1 in the proposition are independent because the fact the forms in (I.24) form a basis of the module of all forms implies in particular that a finite number of them has full rank at all point as vectors in the cotangent vector space. Hence, from the finite dimensional inverse function theorem, one may locally replace, in $x_{1}, \ldots, x_{n}, u_{1}, \ldots, u_{m}, \ldots, u_{1}^{(K)}, \ldots, u_{m}^{(K)}, \widetilde{n}+\widetilde{m}$ coordinates with the functions $z_{1} \circ \varphi, \ldots, z_{\tilde{n}} \circ \varphi, v_{1} \circ \varphi, \ldots, v_{\widetilde{m}} \circ \varphi$. In particular, there exists $n+m$ functions $\xi_{i}$ and $\zeta_{i}^{0}$ defined on a neighborhood of $\left(\bar{z}, \bar{v}, \dot{\bar{v}}, \ldots, \bar{v}^{(L)}\right)$-with $\varphi(\overline{\mathcal{X}})=\left(\bar{z}, \bar{v}, \dot{\bar{v}}, \ddot{\bar{v}}, \ldots, \bar{v}^{(L)}\right)$ - and such that

$$
\begin{array}{ll}
x_{i}=\xi_{i}\left(z \circ \varphi, v \circ \varphi, \ldots, v^{(L)} \circ \varphi, \mathcal{Y}\right) & i=1, \ldots, n \\
u_{i}=\zeta_{i}^{0}\left(z \circ \varphi, v \circ \varphi, \ldots, v^{(L)} \circ \varphi, \mathcal{Y}\right) & i=1, \ldots, m
\end{array}
$$

where $\mathcal{Y}$ represents some of the $n+(K+1) m$ variables $x, u, \dot{u}, \ldots, u^{(K)}$ (all minus $\widetilde{n}+(L+1) \widetilde{m}$ of them). $\mathrm{d} x_{i}$ and $\mathrm{d} u_{i}$ may be computed by differentiating (I.25); the expression involves the partial derivatives of the functions $\xi_{i}$ and $\zeta_{i}$ and comparing with the expressions in (I.24), one may conclude that

$$
\frac{\partial \xi_{i}}{\partial \mathcal{Y}}=0, \frac{\partial \zeta_{i}^{0}}{\partial \mathcal{Y}}=0
$$

and we may write, instead of (I.25),

$$
\begin{array}{lll}
x_{i}=\xi_{i}\left(z \circ \varphi, v \circ \varphi, \ldots, v^{(L)} \circ \varphi\right) & i=1, \ldots, n \\
u_{i}=\zeta_{i}^{0}\left(z \circ \varphi, v \circ \varphi, \ldots, v^{(L)} \circ \varphi\right) & i=1, \ldots, m
\end{array}
$$

We then define the functions $\zeta_{i}^{j}$ for $j>0$ by

$$
\zeta_{i}^{j}=L_{\widetilde{F}} \zeta_{i}^{0}
$$

(note that this makes $\zeta_{i}^{j}$ a smooth function of $\left.z, v, \ldots, v^{(l+j)}\right)$ and we define $\psi$ by

$$
\begin{aligned}
\psi(z, v, \dot{v}, \ddot{v}, \ldots) & =(x, u, \dot{u}, \ddot{u}, \ldots) \\
\text { with } x_{i} & =\xi_{i}\left(z, v, \ldots, v^{(L)}\right) \\
u_{i} & =\zeta_{i}^{0}\left(z, v, \ldots, v^{(L)}\right) \\
\dot{u}_{i} & =\zeta_{i}^{1}\left(z, v, \ldots, v^{(L+1)}\right)
\end{aligned}
$$

Its is straightforward to check that (I.23), (I.28), (I.29) and the fact that $L_{F}^{j} u$ is $u^{(j)}$ imply that $\varphi \circ \psi=I d$ and $\psi \circ \varphi=I d$. 


\section{I.4 Dynamic equivalence}

The objective of the previous sections is the following definition. As announced in the introduction, it mimics the notion of equivalence, or equivalence by endogenous dynamic feedback given in [26] for analytic systems (analyticity plays no role at all in the definition of local equivalence), which coincides with the one given in $[16,17]$ when the transformations are algebraic. The present definition is more concise than in [26] and allows some simple geometric considerations, but the concept of equivalence is the same one. It also coincides with "dynamic equivalence" as defined in [23, 24], see below. It is proved in [26] that if two systems are equivalent in this sense then there exists a dynamic feedback in the sense of (I.2) which is endogenous and nonsingular and transforms one system into a "prolongation" of the other.

Definition I.1 (Equivalence) Two systems $F$ on $\mathcal{M}_{\infty}^{m, n}$ and $\widetilde{F}$ on $\mathcal{M}_{\infty}^{\tilde{m}, \tilde{n}}$ are equivalent at points $\overline{\mathcal{X}} \in \mathcal{M}_{\infty}^{m, n}$ and $\overline{\mathcal{Y}} \in \mathcal{M}_{\infty}^{\tilde{m}, \tilde{n}}$ if and only if there exists a neighborhood $U$ of $\overline{\mathcal{X}}$ in $\mathcal{M}_{\infty}^{m, n}$, a neighborhood $V$ of $\overline{\mathcal{Y}}$ in $\mathcal{M}_{\infty}^{\widetilde{m}, \widetilde{n}}$, and a diffeomorphism $\varphi$ from $U$ to $V$ such that $\varphi(\mathcal{X})=\mathcal{Y}$ and, on $U$,

$$
\widetilde{F}=\varphi_{*} F .
$$

They are globally equivalent if there exists a diffeomorphism $\varphi$ from $\mathcal{M}_{\infty}^{m, n}$ to $\mathcal{M}_{\infty}^{\tilde{m}, \tilde{n}}$ such that (I.30) holds everywhere.

Note that in the definition of local equivalence, the diffeomorphism is only defined locally. This might be worrying : it is not very practical to know that something may be constructed in a region which imposes infinitely many constraints on infinitely many derivatives of the input $u$. This actually does not occur because a neighborhood $U$ of a point $\mathcal{X}$ contains an open set of the form $\pi_{K}^{-1}\left(U_{K}\right)$ with $U_{K}$ open in $\mathcal{M}_{K}^{m, n}$, so that being in $U$ imposes some constraints on $x, u, \dot{u}, \ddot{u}, \ldots, u^{(K)}$ but none on $u^{(K+1)}, u^{(K+2)}, \ldots$.

Some notions of dynamic equivalence ("dynamic equivalence" and "dynamic feedback equivalence") are also given in [23, 24]. To describe them, let us come back to the framework of section I.2, where $\mathcal{M}_{\infty}^{m, n}$ is a sub-bundle of $J^{\infty}(\pi)$ and $\mathcal{M}_{\infty}^{\tilde{m}, \tilde{n}}$ is a sub-bundle of $J^{\infty}(\widetilde{\pi})$; the transformations considered in $[23,24]$ have to be defined from $J^{\infty}(\pi)$ to $J^{\infty}(\widetilde{\pi})$ whereas our diffeomorphism $\varphi$ is only defined on $\mathcal{M}_{\infty}^{m, n}$ (and maps it onto $\mathcal{M}_{\infty}^{\tilde{m}, \widetilde{n}}$ ); actually, Lie-Bäcklund transformations are usually defined, like in [23, 24], all over $J^{\infty}(\pi)$; this is referred to as outer transformations, or outer symmetries if it maps a system into itself, whereas inner transformations are these, like our $\varphi$, defined only "on the solutions", i.e. on $\mathcal{M}_{\infty}^{m, n}$. Since the transformations in [24] are required to be invertible on the solutions only, it is proved there that a transformation like our $\varphi$ may be extended (at least locally) to $J^{\infty}(\pi)$ and therefore that local equivalence in the sense of Definition I.1 is the same as the local version of the one called "dynamic equivalence" (and not "dynamic feedback equivalence") in [24].

It is clear that equivalence is an equivalence relation on systems, i.e. on vector fields of the form (I.19) because the composition of two diffeomorphisms is a diffeomorphism. There is not however a natural group acting on systems since a given diffeomorphism might transform a system $F$ into a system $G$ and transform another system $F^{\prime}$ into a vector field on $\mathcal{M}_{\infty}^{m, n^{\prime}}$ which is not a system. For instance, for $p_{1}, \ldots, p_{m}$ nonnegative, the diffeomorphism $\Upsilon_{n,\left(p_{1}, \ldots, p_{m}\right)}$ defined in (I.15) transforms any system on $\mathcal{M}_{\infty}^{m, n}$ into a system 
on $\mathcal{M}_{\infty}^{m, n+p_{1}+\ldots+p_{m}}$ whereas the diffeomorphism $\Upsilon_{n+p_{1}+\ldots+p_{m},\left(-p_{1}, \ldots,-p_{m}\right)}$-its inverse- transforms most systems on $\mathcal{M}_{\infty}^{m, n+p_{1}+\ldots+p_{m}}$ into a vector field on $\mathcal{M}_{\infty}^{m, n}$ which is not a "system" because it does not have the required structure on the coordinates which are called "inputs" on $\mathcal{M}_{\infty}^{m, n}$. Two important questions arise : what is exactly the class of diffeomorphisms which transform at least one system into another system and what is the class of vector fields equivalent to a system by such a diffeomorphism. An element of answer to the latter question is that "non-classical" systems $[13,10]$, i.e. these where the right-hand side of (I.1) depends also on some time-derivatives of $u$, or vector fields on which the second constraint in (I.21) does not hold, are in this class of vector fields because they are transformed by $\Upsilon_{n,(K, \ldots, K)}$, where $K$ is the number of derivatives of the input appearing in the system, into a (classical) system, this illustrates that generalized state-space representations [13, 10] are "natural"; however, it is clear that the class of vector fields which may be conjugated to a "system" is much larger: the only system (classical or not) on $\mathcal{M}_{\infty}^{m, 0}$ is $C$ and very few systems on $\mathcal{M}_{\infty}^{m, n}$ are transformed into $C$ by $\Upsilon_{n,(-n, 0, \ldots, 0)}$ for example. A partial answer to the former question is given by :

Theorem I.1 The number of inputs $m$ is invariant under equivalence.

Proof : For any function $h, L_{\widetilde{F}}\left(h \circ \varphi^{-1}\right)=\left(L_{F} h\right) \circ \varphi^{-1}$. The integer $m$ from Proposition I.2 is therefore preserved by a diffeomorphism $\varphi$.

Further remarks on the class of diffeomorphisms which transform at least one system into another system may be done. One may restrict its attention to systems of the same dimension, i.e. to diffeomorphisms from $\mathcal{M}_{\infty}^{m, n}$ to itself because if $\varphi$ goes from $\mathcal{M}_{\infty}^{m, n}$ to $\mathcal{M}_{\infty}^{m, N}$ with $N>n$ and transforms a system into a system, $\Upsilon_{n,(N-n, 0, \ldots, 0)} \circ \varphi$ is a diffeomorphism of $\mathcal{M}_{\infty}^{m, N}$ that transforms a system into a system. In the single-input case $(m=1)$, as stated in section I.6, $\varphi$ must be static, which is a complete answer to the question because a static diffeomorphism transforms any system into a system. In the case of at least two inputs $(m>1)$, the literature ([25, Theorem 4.4.5] or [1, Theorem 3.1], but these have to be adapted since they are stated in an "outer" context) tells us that either $\varphi$ is static or it does not preserve the fibers of $\pi_{k}: \mathcal{M}_{\infty}^{m, n} \rightarrow \mathcal{M}_{k}^{m, n}$ for any $k$, i.e., if $\varphi$ is given by $\varphi(x, u, \dot{u}, \ddot{u}, \ldots)=(z, v, \dot{v}, \ddot{v}, \ldots)$, there is no $k$ such that $\left(z, v, \dot{v}, \ldots, v^{(k)}\right)$ is a function of $\left(x, u, \dot{u}, \ldots, u^{(k)}\right)$ only. This is related to the statement [7] that, when dynamic feedback is viewed as adding some integrators plus performing a static feedback, it is inefficient to add the same number of integrators on each input.

\section{I.5 Static equivalence}

Definition I.2 (Static equivalence) Two systems $F$ on $\mathcal{M}_{\infty}^{m, n}$ and $\widetilde{F}$ on $\mathcal{M}_{\infty}^{\tilde{m}, \tilde{n}}$ are (locally/globally) static equivalent if and only if they are (locally/globally) feedback equivalent with the diffeomorphism $\varphi$ in (I.30) being a static diffeomorphism.

From Proposition I.1, we know that a static diffeomorphism really defines an invertible static feedback transformation in the usual sense, this is summed up in the following :

Theorem I.2 Both the number of inputs $m$ and the dimension $n$ of the state are invariant under static equivalence. Moreover, $\pi_{-1} \circ \varphi$ provides a local diffeomorphism in the classical 
state-space $\mathbb{R}^{n}$ and the $u$ component of $\pi_{0} \circ \varphi$ provides a nonsingular feedback transformation which together provide an invertible static feedback transformation in the usual sense.

\section{I.6 The single-input case}

It was proved in $[7,6]$ that a single-input system which is "dynamic feedback linearizable" is "static feedback linearizable". The meaning of dynamic feedback linearizable was weaker that being equivalent to a linear system as meant here : "exogenous" feedbacks (see [26]) were allowed in [7] as well as singular (feedbacks which may change the number of inputs for example). The following Theorem I.3 may be viewed as a generalization of this result to non-linearizable systems, but with a more restrictive dynamic equivalence.

It is known that the only transformations on an infinite jet bundle with only one "dependent variable" which preserves the contact structure (Lie-Bäcklund transformation in [1], $\mathcal{C}$-transformation in [25]) are infinite prolongations of transformations on first jets (Lie transformation according to [25]), see for instance [25, Theorems 6.3.7 and 4.4.5]. The following result is similar in spirit. We give the full proof, a little long but elementary : it basically consists in counting the dimensions carefully, it is complicated by the fact that we do not make any a priori regularity assumption (for instance, the functions $\chi_{i}$ and $\psi_{i}$ defining the diffeomorphism are not assumed to depend on a locally constant number of derivatives of $u$ ).

Theorem I.3 Let $F$ and $\widetilde{F}$ be two systems on $\mathcal{M}_{\infty}^{1, n}$ (i.e. two single input systems with the same number of states). Any (local/global) diffeomorphism $\varphi$ such that $\widetilde{F}=\varphi_{*} F$ is static. Hence they are (locally/globally) equivalent if and only if they are (locally/globally) static equivalent.

Proof : The second statement is a straightforward consequence of the first one. Let us consider a diffeomorphism $\varphi$ such that $\widetilde{F}=\varphi_{*} F$ and prove that $\varphi$ is static. Suppose that, in coordinates, $\varphi$ and $\varphi^{-1}$ are given by $\varphi(x, \mathcal{U})=(z, \mathcal{V})$ and $\varphi^{-1}(z, \mathcal{V})=(x, \mathcal{U})$ with :

$$
\begin{aligned}
z & =\chi_{-1}(x, \mathcal{U}) & x & =\psi_{-1}(z, \mathcal{V}) \\
v & =\chi_{0}(x, \mathcal{U}) & u & =\psi_{0}(z, \mathcal{V}) \\
& \vdots & & \vdots \\
v^{(j)} & =\chi_{j}(x, \mathcal{U}) & u^{(j)} & =\psi_{j}(z, \mathcal{V}) \\
& \vdots & & \vdots
\end{aligned}
$$

Since $\widetilde{F}=\varphi_{*} F$, we have

$$
\begin{aligned}
L_{F} \chi_{-1}(x, \mathcal{U}) & =\widetilde{f}\left(\chi_{-1}(x, \mathcal{U}), \chi_{0}(x, \mathcal{U})\right) \\
L_{F} \chi_{j}(x, \mathcal{U}) & =\chi_{j+1}(x, \mathcal{U}) \text { for } j \geq 0
\end{aligned}
$$

Let $\mathcal{X}$ be an arbitrary point of the domain where $\varphi$ is defined. From the definition of a diffeomorphism, there is an integer $J \geq-1$ and a neighborhood $U$ of $\mathcal{X}\left(J\right.$ is $\delta\left(\pi_{0} \circ \varphi\right)(\mathcal{X})$ if $U$ is small enough) such that $\chi_{-1}$ and $\chi_{0}$ depend only on $x, u, \dot{u}, \ldots, u^{(J)}$ on $U$ and $\frac{\partial \chi_{-1}}{\partial u^{(J)}}$ and $\frac{\partial \chi_{0}}{\partial u^{(J)}}$ are not both identically zero on $U$ (one might take all the open set where $\varphi$ is defined $-\mathcal{M}_{\infty}^{m, 1}$ in the global case- instead of $U$, but this might cause $J$ to be infinite). 
If $J$ was $-1, \chi_{-1}$ and $\chi_{0}$ would both depend only on $x$, but the dimension of $x$ is $n$ and the dimension of $\left(\chi_{-1}, \chi_{0}\right)$ is $n+1$ : there would be a function such that $h\left(\chi_{-1}, \chi_{0}\right)$ would be zero on $U$ and this would prevent $\varphi$ from being a diffeomorphism; hence $J \geq 0$.

The first equation in (I.32), and the second one for $j=0$, imply :

$$
\begin{aligned}
\frac{\partial \chi_{-1}}{\partial x} f(x, u)+\frac{\partial \chi_{-1}}{\partial u} \dot{u}+\cdots+\frac{\partial \chi_{-1}}{\partial u^{(J)}} u^{(J+1)} & =\tilde{f}\left(\chi_{-1}\left(x \ldots u^{(J)}\right), \chi_{0}\left(x \ldots u^{(J)}\right)\right), \\
\frac{\partial \chi_{0}}{\partial x} f(x, u)+\frac{\partial \chi_{0}}{\partial u} \dot{u}+\cdots+\frac{\partial \chi_{0}}{\partial u^{(J)}} u^{(J+1)} & =\chi_{1}(x, \mathcal{U}) .
\end{aligned}
$$

By taking the derivative with respect to $u^{(J+1)}$ of the first equation and with respect to $u^{(j)}$ for $j \geq J+2$ of the second equation,

$$
\frac{\partial \chi_{-1}}{\partial u^{(J)}}=0 \text { and } \quad 0=\frac{\partial \chi_{1}}{\partial u^{(j)}} \quad \text { for } j \geq J+2 .
$$

This implies that that $\chi_{-1}$ is a function of $x, u, \ldots, u^{(J-1)}(x$ if $J=0)$ only, $\chi_{0}$ is a function of $x, u, \ldots, u^{(J-1)}, u^{(J)}$ only (by definition of $J$ ), and $\chi_{1}$ of $x, u, \ldots, u^{(J-1)}, u^{(J)}, u^{(J+1)}$ only. It is then easy to deduce by induction from the second relation in (I.32) that for all $j \geq 0$, $\chi_{j}$ is a function of $x, u, \ldots, u^{(J+j+1)}$ on this neighborhood with

$$
\frac{\partial \chi_{j}}{\partial u^{(J+j)}}=\frac{\partial \chi_{0}}{\partial u^{(J)}}, \quad j \geq 0
$$

From the first relation in (I.33) and the definition of $J, \frac{\partial \chi_{0}}{\partial u^{(J)}}$ is not identically zero on $U$. Hence, there is a point $\overline{\mathcal{X}}=(\bar{x}, \bar{u}, \dot{\bar{u}}, \ldots) \in U$ such that $\frac{\partial \chi_{0}}{\partial u^{(J)}}(\overline{\mathcal{X}})=\frac{\partial \chi_{0}}{\partial u^{(J)}}\left(\bar{x}, \bar{u}, \ldots, \bar{u}^{(J)}\right) \neq 0$. Let $K$ be $\delta\left(\pi_{0} \circ \varphi^{-1}\right)(\overline{\mathcal{X}})$ - note that it might be smaller than $\delta\left(\pi_{0} \circ \varphi^{-1}\right)(\mathcal{X})$ - i.e. $\psi_{-1}$ and $\psi_{0}$ locally depend only on $z, v, \ldots, v^{(K)}$, and $\frac{\partial \psi_{-1}}{\partial v^{(K)}}$ and $\frac{\partial \psi_{0}}{\partial v^{(K)}}$ are not both identically zero on any neighborhood of $\overline{\mathcal{X}}$. This implies, since $\frac{\partial \chi_{0}}{\partial u^{(J)}}$ is nonzero at $\overline{\mathcal{X}}$, that there is a neighborhood $\bar{U}$ of $\overline{\mathcal{X}}$ such that, on $\bar{U}, \frac{\partial \chi_{0}}{\partial u^{(J)}}$ does not vanish, $\psi_{-1}$ and $\psi_{0}$ depend only on $z, v, \ldots, v^{(K)}$ and $\frac{\partial \psi_{-1}}{\partial v^{(K)}}$ and $\frac{\partial \psi_{0}}{\partial v^{(K)}}$ are not both identically zero. We have, on $\bar{U}$,

$$
\begin{aligned}
& x=\psi_{-1}\left(\chi_{-1}\left(x, u, \ldots, u^{(J-1)}\right), \chi_{0}\left(x, u, \ldots, u^{(J)}\right), \ldots, \chi_{K}\left(x, u, \ldots, u^{(J+K)}\right)\right) \\
& u=\psi_{0}\left(\chi_{-1}\left(x, u, \ldots, u^{(J-1)}\right), \chi_{0}\left(x, u, \ldots, u^{(J)}\right), \ldots, \chi_{K}\left(x, u, \ldots, u^{(J+K)}\right)\right) .
\end{aligned}
$$

$K$ cannot, for the same dimensional reasons as $J$, be equal to -1 , hence $K \geq 0$. Now, suppose that $J \geq 1$. Then $J+K \geq 1$, and taking the derivative of both identities in (I.35) with respect to $u^{(J+K)}$ therefore yields

$$
\frac{\partial \psi_{-1}}{\partial v^{(K)}} \frac{\partial \chi_{K}}{\partial u^{(J+K)}}=\frac{\partial \psi_{0}}{\partial v^{(K)}} \frac{\partial \chi_{K}}{\partial u^{(J+K)}}=0
$$

identically on $\bar{U}$. This is impossible because on one hand $\frac{\partial \chi_{K}}{\partial u^{(J+K)}}$ does not vanish because of (I.34) and on the other hand $K$ has been defined so that $\frac{\partial \psi_{-1}}{\partial v^{(K)}}$ and $\frac{\partial \psi_{0}}{\partial v^{(K)}}$ are not both identically zero on $\bar{U}$. Hence $J \geq 1$ is impossible.

We have proved that $J=0$. Hence $\chi_{j}$ depends only on $x, u \ldots u^{(j)}(x$ for $j=-1)$ for all $j \geq-1$ (see above) and $\frac{\partial \chi_{j}}{\partial u^{(j)}}$ is, for all $j$, nonsingular at all points (consequence of the smooth invertibility of $\varphi$ ). This is the definition of a static diffeomorphism. 


\section{I.7 Dynamic linearization}

$A$ controllable linear system is a system of the form (I.19) where the function $f$ is linear, i.e. $f(x, u)=A x+B u$ with $A$ and $B$ constant matrices, and (Kalman rank condition) the rank of the columns of $B, A B, A^{2} B$ is $n$.

There is a canonical form under static feedback, known as Brunovský canonical form [5] for these systems : they may be transformed via a static diffeomorphism (from $\mathcal{M}_{\infty}^{m, n}$ to itself) to a linear system where $A$ and $B$ have the form of some "chains of integrators" of "length" $r_{1}, \ldots, r_{m}$; the diffeomorphism $\Upsilon_{n,\left(-r_{1}, \ldots,-r_{m}\right)}$ from $\mathcal{M}_{\infty}^{m, n}$ to $\mathcal{M}_{\infty}^{m, 0}$ (see (I.15)) which "cuts off" all these integrators then transforms this system into $C$ (see (I.20)) :

Proposition I.4 ([5]) A controllable linear system with $m$ inputs is globally equivalent to the canonical system $C$ on $\mathcal{M}_{\infty}^{m, 0}$.

We wish to call dynamic linearizable a system which is equivalent to a controllable linear system. From the above proposition, this may equivalently be stated as :

Definition I.3 A system is (locally/globally) dynamic linearizable if and only if it is (locally/globally) equivalent to the canonical linear system $C$ on $\mathcal{M}_{\infty}^{0, m}$.

Of course this concept is the same as in [26, "analytic approach"] since the equivalence is the same. In $[16,17,26]$, the notion of linearizing outputs or flat outputs is used to define flat control systems as these which admit such outputs. It is proved that flatness coincides with equivalence by endogenous feedback to a controllable linear system. In [23, 24] a system is called free if the differential algebra $\left(\mathcal{C}^{\infty}\left(\mathcal{M}_{\infty}^{m, n}\right), L_{F}\right)$ is free; the linearizing outputs we define below are free generators of this differential algebra. The following theorem in a sense re-states the result "flat $\Leftrightarrow$ linearizable by endogenous feedback".

Theorem I.4 (linearizing outputs) A system $F$ on $\mathcal{M}_{\infty}^{m, n}$ is locally dynamic linearizable at a point $\mathcal{X}$ if and only if there exist $m$ smooth functions $h_{1}, \ldots, h_{m}$ from a neighborhood of $\mathcal{X}$ in $\mathcal{M}_{\infty}^{m, n}$ to $\mathbb{R}$ such that $\left(L_{F}^{j} h_{k}\right)_{1 \leq k \leq m, 0 \leq j}$ is a system local of coordinates at $\mathcal{X}$. It is globally dynamic linearizable and only if there exist $m$ smooth functions $h_{1}, \ldots, h_{m}$ from $\mathcal{M}_{\infty}^{m, n}$ to $\mathbb{R}$ such that $\left(L_{F}^{j} h_{k}\right)_{1 \leq k \leq m, 0 \leq j}$ is a global system of coordinates. These functions are called linearizing outputs.

Proof : If $F$ is dynamic linearizable, there exists a (local/global) diffeomorphism $\varphi$ from $\mathcal{M}_{\infty}^{m, n}$ to $\mathcal{M}_{\infty}^{m, 0}$ such that $C=\varphi_{*} F$. Define $h_{k}$ by $h_{k}=v_{k}^{(j)} \circ \varphi$ with $v_{k}^{(j)}$ the canonical coordinates on $\mathcal{M}_{\infty}^{0, m}$. Since $v_{k}^{(j)}=L_{C}^{j} v_{k}$ (the $j$ th Lie derivative of $v_{k}$ along $C$ ) and $C=\varphi_{*} F$, we have

$$
L_{F}^{j} h_{k}=L_{F}^{j}\left(v_{k} \circ \varphi\right)=\left(L_{\varphi_{*} F}^{j} v_{k}\right) \circ \varphi=v_{k}^{(j)} \circ \varphi
$$

so that, since $\varphi$ is a diffeomorphism and $\left(v_{k}^{(j)}\right)_{1 \leq k \leq m, 0 \leq j}$ is a system of coordinates on $\mathcal{M}_{\infty}^{m, 0}$, $\left(v_{k}^{(j)} \circ \varphi\right)_{1 \leq k \leq m, 0 \leq j}$ is a system of coordinates on $\mathcal{M}_{\infty}^{m, n}$. Conversely, if there exist $m$ functions $h_{1}, \ldots, h_{m}$ enjoying this property, then one may define the diffeomorphism $\varphi$ mapping a point $(x, \mathcal{U})$ of $\mathcal{M}_{\infty}^{m, n}$ to the point of $\mathcal{M}_{\infty}^{0, m}$ whose coordinate $v_{k}^{(j)}$ is $L_{F}^{j} h_{k}(x, \mathcal{U})$. It is clear that $\varphi_{*} F=C$. 
Of course, this is far from being a solution to dynamic feedback linearization since one has to determine if linearizing outputs exist, which is not an easy task; see Chapter II for bibliography and a discussion of this topic. Let us give a rather convenient way of tackling this problem by transforming it into its "infinitesimal" version. Recall that a Pfaffian system is a family of differential forms of degree 1 with constant rank; any family of forms generating the same module (or co-distribution) defines the same Pfaffian system. The infinitesimal version of linearizing outputs is and object already defined $n[2,33]$ :

Definition I.4 A Pfaffian system $\left(\omega_{1}, \ldots, \omega_{m}\right)$ is called a linearizing Pfaffian system at point $\mathcal{X}$ if and only if, for a certain neighborhood $U$ of $\mathcal{X}$, the restriction to $U$ of the forms $L_{F}^{j} \omega_{k}, j \geq 0,1 \leq k \leq m$ form a basis of the $\mathcal{C}^{\infty}(U)$-module $\Lambda^{1}(U)$ of all differential forms on $U$.

We have three comments on this definition. Firstly, this is a property of the Pfaffian system $\left(\omega_{1}, \ldots, \omega_{m}\right)$ rather than the $m$-uple of 1 -forms since it is not changed when changing the collection of forms $\omega_{1}, \ldots, \omega_{m}$ into another collection which span the same module. Secondly, one may prove than the rank of such a Pfaffian system must be $m$ (see the proof of Proposition I.2). Finally, one should not be mislead by the terminology : existence of a linearizing Pfaffian system does not imply linearizability :

Theorem I.5 A system $F$ on $\mathcal{M}_{\infty}^{m, n}$ is locally dynamic linearizable at point $\mathcal{X}$ if and only if there exists, on a neighborhood of $\mathcal{X}$, a linearizing Pfaffian system $\left(\omega_{1}, \ldots, \omega_{m}\right)$ which is locally completely integrable.

By locally completely integrable, we mean the classical Frobenius condition $d \omega_{k} \wedge \omega_{1} \wedge$ $\ldots \wedge \omega_{m}=0$; note that the condition that $\left(L_{F}^{j} \omega_{k}\right)_{1 \leq k \leq m, 0 \leq j}$ be a basis of $\Lambda^{1}(U)$ implies that the rank at all point of $\left(\omega_{1}, \ldots, \omega_{m}\right)$ is $m$, and is therefore constant.

Proof : The condition is obviously necessary from Theorem I.4 by taking $\omega_{k}=\mathrm{d} h_{k}$. Conversely, one may apply the finite dimensional Frobenius theorem to $\left(\omega_{1}, \ldots, \omega_{m}\right)$ because they depend on a finite number of variables, and, as noticed above, they have constant rank $m$ : there exists $m$ functions $h_{1} \ldots h_{m}$ (of the same number of variables than these appearing in $\left.\omega_{1} \ldots \omega_{m}\right)$ such that $\mathrm{d} h_{1}, \ldots, \mathrm{d} h_{m}$ span the same co-distribution than $\omega_{1}, \ldots, \omega_{m}$; this implies that $\left(L_{F}^{j} \mathrm{~d} h_{k}\right)_{1 \leq k \leq m, 0 \leq j}$ is also a basis of $\Lambda^{1}(U)$. Define the map $\varphi: U \rightarrow \mathcal{M}_{\infty}^{m, 0}$ as assigning to a point $(x, \mathcal{U})$ of $\mathcal{M}_{\infty}^{m, n}$ to the point of $\mathcal{M}_{\infty}^{0, m}$ whose coordinate $v_{k}^{(j)}$ is $L_{F}^{j} h_{k}(x, \mathcal{U})$. It is clear that for all function $\tilde{h} \in \mathcal{C}^{\infty}\left(\mathcal{M}_{\infty}^{0, m}\right),\left(L_{C} \tilde{h}\right) \circ \varphi=L_{\varphi}(\tilde{h} \circ \varphi)$, so that theorem I.3 implies that $\varphi$ is a local diffeomorphism.

This result is more interesting in the light of the fact that a controllable system admits a linearizing Pfaffian system at "almost all" points. Next chapter develops further this point of view, see also [2] for a more algebraic approach.

\section{Acknowledgments}

After presenting the present material at a meeting of the "GdR Automatique" (CNRS), the author had some discussions with M. Fliess, J. Lévine, P. Rouchon and P. Martin and discovered they had recently developed a very similar though independent approach. He wishes to thank them for these interesting discussions and for communicating to him the preprints $[18,19]$. 


\title{
Chapter II
}

\section{Infinitesimal Brunovský form for nonlinear systems with applications to dynamic linearization}

\author{
Eduardo Aranda-Bricaire, Claude H. Moog and Jean-Baptiste Pomet
}

\begin{abstract}
We define, in an infinite-dimensional differential geometric framework, the "infinitesimal Brunovský form" which we previously introduced in another framework and link it with equivalence via diffeomorphism to a linear system, which is the same as linearizability by "endogenous dynamic feedback".
\end{abstract}

Keywords : Nonlinear control systems, Dynamic feedback linearization, endogenous dynamic feedback, Brunovský Canonical form, Pfaffian systems, flat systems, linearized control system.

\section{II.1 Introduction and Problem Statement}

The purpose of this note is to present a "geometric" version of the constructions made in $[2,33]$. The framework from Chapter I will be used ; it is briefly summed up in section II.2.

The contribution of $[2,33]$ was to construct a so-called "infinitesimal Brunovský form" ("non-exact Brunovský form" in [33]) for controllable nonlinear systems and to relate it to dynamic linearization; they use the linear algebraic framework introduced in [11]. The point of view on the feedback linearization problem was the one of looking for "linearizing outputs", following the idea of $[16,17,26]$. It is therefore, following the terms of $[16,17,26]$, linearization via endogenous dynamic feedback. In [33], we relied explicitly upon the notion of differential flatness $[16,17,26]$, whereas [2] re-defines the notion of linearizing outputs in terms of dynamic decoupling and structure at infinity.

Here, in the framework of Chapter I, dynamic linearization is equivalence to a linear system via diffeomorphism on the extended state space manifold; linearizing outputs are 
functions such that these and all their "time-derivatives" are a set of local coordinates on the generalized state-space manifold. The main interest of this approach over the algebraic ones is that it is possible to give local notions, and therefore singularities are not ignored.

In section II.3, we define the infinitesimal Brunovský form and relate it to some work on time-varying linear systems and linearized systems of nonlinear systems $[14,15]$. In section II.4, we relate this construction to existence of linearizing outputs, and explain why it provides a good framework for searching linearizing outputs.

\section{II.2 Summary of Chapter I}

2.1. The "infinite dimensional manifold" $\mathcal{M}_{\infty}^{m, n}$ is, for short, $\mathbb{R}^{n} \times\left(\mathbb{R}^{m}\right)^{\mathbb{N}}$. A global system of coordinates is $x_{1}, \ldots, x_{n}, u_{1}, \ldots, u_{m}, \dot{u}_{1}, \ldots, \dot{u}_{m}, \ddot{u}_{1}, \ldots$ It is endowed with the product topology : an open set may be described by some restrictions on a finite number of coordinates, i.e. there is a $\tilde{k}$ such that, considered as an open set of $\mathbb{R}^{n} \times\left(\mathbb{R}^{m}\right)^{\mathbb{N}}=$ $\mathbb{R}^{n} \times\left(\mathbb{R}^{m}\right)^{\tilde{k}} \times\left(\mathbb{R}^{m}\right)^{\mathbb{N}}$, it can be written $\widetilde{O} \times\left(\mathbb{R}^{m}\right)^{\mathbb{N}}$ with $\widetilde{O}$ an open set of $\mathbb{R}^{n} \times\left(\mathbb{R}^{m}\right)^{\tilde{k}}$.

2.2. A smooth function on $\mathcal{M}_{\infty}^{m, n}$ is one which depends only on a finite number of coordinates and is smooth as a function of these coordinates. $\mathcal{C}^{\infty}(U)$ stands for the algebra of smooth functions defined on an open subset $U$ of $\mathcal{M}_{\infty}^{m, n}$. A smooth mapping from $\mathcal{M}_{\infty}^{m, n}$ to $\mathcal{M}_{\infty}^{\tilde{m}, \tilde{n}}$ is a mapping whose composition with any smooth function is a smooth function. A diffeomorphism from $\mathcal{M}_{\infty}^{m, n}$ to $\mathcal{M}_{\infty}^{\widetilde{m}, \tilde{n}}$ is a bijective smooth mapping whose inverse is a smooth mapping.

2.3. A vector field is a possibly infinite linear combination $\sum v_{i} \frac{\partial}{\partial w_{i}}$ where the $v_{i}$ 's are smooth functions and the $w_{i}$ 's are some of the coordinates $x_{1}, \ldots, x_{n}, u_{1}, \ldots, u_{m}, \dot{u}_{1}, \ldots$, $\dot{u}_{m}, \ldots$ A differential form of degree 1 (or 1-form) is, with the same conventions, a finite linear combination $\sum v_{i} \mathrm{~d} w_{i} . \Lambda^{1}(U)$ stands for the $\mathcal{C}^{\infty}(U)$-module of 1 -forms defined on $U$.

2.4. All the "formulas" from finite dimensional differential calculus involving objects like Lie brackets and Lie derivatives are valid. For instance, the Lie derivative of a form $\omega=\sum v_{i} \mathrm{~d} w_{i}$ along a vector field $F$ may be computed, in coordinates, according to $L_{F} \omega=\sum L_{F} v_{i} \mathrm{~d} w_{i}+$ $v_{i} \mathrm{~d}\left(L_{F} w_{i}\right)$. Also, a diffeomorphism carries vector fields or differential forms from one manifold to another, we use the usual notation $\varphi_{*} F$ or $\varphi^{*} \omega$.

2.5. A smooth control system (I.1) :

$$
\dot{x}=f(x, u)
$$

with state $x \in \mathbb{R}^{n}$ and input $u \in \mathbb{R}^{m}$ is represented by a single vector field

$$
F=f(x, u) \frac{\partial}{\partial x}+\dot{u} \frac{\partial}{\partial u}+\ddot{u} \frac{\partial}{\partial \dot{u}}+\ldots
$$

on $\mathcal{M}_{\infty}^{m, n}$. We often refer to "system $F$ ", confusing system (II.1) with vector field $F$.

2.6. The Lie derivative along $F$ defined by (II.2) is simply the "time-derivative" according to (II.1) : we often write $\dot{\varphi}$ or $\dot{\omega}$ instead of $L_{F} \varphi$ or $L_{F} \omega$ for a function $\varphi$ or a 1 -form $\omega$. 
2.7. A diffeomorphism from $\mathcal{M}_{\infty}^{m, n}$ to $\mathcal{M}_{\infty}^{\tilde{m}, \tilde{n}}$ given by $(x, u, \dot{u}, \ddot{u}, \ldots) \mapsto(z, v, \dot{v}, \ddot{v}, \ldots)$ is said to be a static diffeomorphism if and only if $z$ depends only on $x, v$ depends only on $x$ and $u, \dot{v}$ depends only on $x, u$ and $\dot{u} \ldots$ A static diffeomorphism is nothing more than a nonsingular static transformation in the usual sense : if $F$ is a system on $\mathcal{M}_{\infty}^{m, n}$ and $\widetilde{F}$ is a system on $\mathcal{M}_{\infty}^{\widetilde{m}, \tilde{n}}$, existence of a static diffeomorphism $\varphi$ such that $\widetilde{F}=\varphi_{*} F$ is equivalent to $n=\tilde{n}, m=\tilde{m}$ and static equivalence of the control systems associated with $F$ and $\widetilde{F}$.

2.8. Of course, $n=0$ is not ruled out in the above definitions, coordinates on $\mathcal{M}_{\infty}^{m, 0}$ are simply $\{u, \dot{u}, \ddot{u}, \ldots\}$, and the only system is the canonical linear system with $m$ inputs (I.20) :

$$
C=\sum_{0}^{\infty} u^{(j+1)} \frac{\partial}{\partial u^{(j)}} .
$$

It has "no state", but one should not worry about this since $n=0$ is obtained after "cutting all the integrators" in a canonical linear system [5] and arbitrarily renaming some states "inputs". Dynamic linearizability is conjugation via a diffeomorphism to system $C$ :

Definition II.1 (rephrasing of Definition I.3) A system $F$ is locally dynamic linearizable at point $\mathcal{X} \in \mathcal{M}_{\infty}^{m, n}$ if and only if there exists a neighborhood $U$ of $\mathcal{X}$ in $\mathcal{M}_{\infty}^{m, n}$, an open subset $V$ of $\mathcal{M}_{\infty}^{m, 0}$, and a diffeomorphism $\varphi$ from $U$ to $V$ such that, on $U, \quad \varphi_{*} F=C$.

2.9. Consider a $\mathcal{C}^{\infty}(U)$-module of vector fields $\mathcal{D}$ (resp. of forms $\mathcal{H}$ ), defined on an open set $U$. The annihilator of $\mathcal{D}$ is the module of the forms which vanish on all the vector fields of $\mathcal{D}$, and vice-versa :

$$
\mathcal{H}^{\perp}=\{X, \forall \omega \in \mathcal{H},\langle\omega, X\rangle=0\} ; \quad \mathcal{D}^{\perp}=\{\omega, \forall X \in \mathcal{D},\langle\omega, X\rangle=0\} .
$$

$\mathcal{D}(\mathcal{X})($ resp. $\mathcal{H}(\mathcal{X}))$ denotes the subspace of the tangent (resp. cotangent) space to $\mathcal{M}_{\infty}^{m, n}$ at point $\mathcal{X} \in U$ made of all the $X(\mathcal{X})$ for $X \in \mathcal{D}$ (resp. $\omega(\mathcal{X})$ for $\omega \in \mathcal{H})$. We call the dimension of $\mathcal{D}(\mathcal{X})($ resp. $\mathcal{H}(\mathcal{X}))$ the pointwise rank of $\mathcal{D}$ (resp. $\mathcal{H}$ ) at point $\mathcal{X}$. $\mathcal{D}$ or $\mathcal{H}$ is said to be nonsingular at point $\mathcal{X}$ if and only its the pointwise rank is constant in a neighborhood of $\mathcal{X}$; it is then equal to the rank of the module over $\mathcal{C}^{\infty}(U)$.

\section{II.3 The Infinitesimal Brunovský Form}

Let us define the following sequence of $\mathcal{C}^{\infty}\left(\mathcal{M}_{\infty}^{m, n}\right)$-modules of vector fields :

$$
\begin{aligned}
\mathcal{D}_{-j} & =\operatorname{Span}\left\{\frac{\partial}{\partial u^{(j+1)}}, \frac{\partial}{\partial u^{(j+2)}}, \ldots\right\} \quad j \geq 0 \\
& \vdots \\
\mathcal{D}_{0} & =\operatorname{Span}\left\{\frac{\partial}{\partial \dot{u}}, \frac{\partial}{\partial \ddot{u}}, \frac{\partial}{\partial u^{(3)}}, \ldots\right\} \\
\mathcal{D}_{1} & =\operatorname{Span}\left\{\frac{\partial}{\partial u}, \frac{\partial}{\partial \dot{u}}, \frac{\partial}{\partial \ddot{u}}, \frac{\partial}{\partial u^{(3)}}, \ldots\right\} \\
& \vdots \\
\mathcal{D}_{k+1} & =\mathcal{D}_{k}+\left[F, \mathcal{D}_{k}\right] \\
& \vdots \\
\mathcal{D}_{\infty} & =\sum_{k} \mathcal{D}_{k}
\end{aligned}
$$


and, since these are "infinite-dimensional", we define for each $\mathcal{D}_{k}(k \geq 1)$ its " $\frac{\partial}{\partial x}$ part" :

$$
\widehat{\mathcal{D}}_{k}=\mathcal{D}_{k} \cap \operatorname{Span}\left\{\frac{\partial}{\partial x}\right\}, \quad k \in[1, \infty]
$$

$\left(\operatorname{Span}\left\{\frac{\partial}{\partial x}\right\}\right.$ stands for the $\mathcal{C}^{\infty}\left(\mathcal{M}_{\infty}^{m, n}\right)$-module generated by $\left.\frac{\partial}{\partial x_{1}}, \ldots, \frac{\partial}{\partial x_{n}}\right)$, which makes $\widehat{\mathcal{D}}_{k}(\mathcal{X})$ (see paragraph 2.9) finite-dimensional for all $\mathcal{X} \in \mathcal{M}_{\infty}^{m, n}$ ), and yields

$$
\mathcal{D}_{k}=\widehat{\mathcal{D}}_{k} \oplus \mathcal{D}_{1}, \quad k \in[1, \infty] .
$$

Note that (II.5) and (II.6) are both valid for $k=\infty$ and that $\widehat{\mathcal{D}}_{\infty}$ might as well have been defined by $\widehat{\mathcal{D}}_{\infty}=\sum_{k} \widehat{\mathcal{D}}_{k}$. We define also a sequence of $\mathcal{C}^{\infty}\left(\mathcal{M}_{\infty}^{m, n}\right)$-modules of forms :

$$
\begin{aligned}
\mathcal{H}_{-j} & =\operatorname{Span}\left\{\mathrm{d} x, \mathrm{~d} u, \ldots, \mathrm{d} u^{(j)}\right\} \quad j \geq 0 \\
& \vdots \\
\mathcal{H}_{0} & =\operatorname{Span}\{\mathrm{d} x, \mathrm{~d} u\} \\
\mathcal{H}_{1} & =\operatorname{Span}\{\mathrm{d} x\} \\
& \vdots \\
\mathcal{H}_{k+1} & =\left\{\omega \in \mathcal{H}_{k}, \dot{\omega}=L_{F} \omega \in \mathcal{H}_{k}\right\} \\
& \vdots \\
\mathcal{H}_{\infty} & =\bigcap_{k} \mathcal{H}_{k} .
\end{aligned}
$$

See paragraphs 2.4 and 2.6 for a definition of $\dot{\omega}$ or $L_{F} \omega$. We have the following relation between the $\mathcal{D}_{k}$ 's and the $\mathcal{H}_{k}$ 's :

Proposition II.1 All the modules $\mathcal{D}_{k}$ and $\mathcal{H}_{k}$ are invariant by static feedback, i.e. by static diffeomorphism of $\mathcal{M}_{\infty}^{m, n}$ (see paragraph 2.7), and, for all $k$,

$$
\mathcal{H}_{\infty} \subset \mathcal{H}_{k+1} \subset \mathcal{H}_{k} \quad, \quad \mathcal{D}_{k} \subset \mathcal{D}_{k+1} \subset \mathcal{D}_{\infty}, \quad \mathcal{H}_{k}=\mathcal{D}_{k}^{\perp}, \quad \mathcal{D}_{k} \subset \mathcal{H}_{k}^{\perp},
$$

with $\mathcal{H}_{k}^{\perp}=\mathcal{D}_{k}$ at points where $\widehat{\mathcal{D}}_{k}$ is nonsingular (see paragraph 2.9).

Proof : From (II.4) and Proposition I.1, a static diffeomorphism $\varphi$ does not change $\mathcal{D}_{k}$ for $k \leq 1$; since the recursive definition of $\mathcal{D}_{k}$ for larger $k$ only uses Lie brackets, it is then clear that the modules built according to (II.4) from $\varphi_{*} F$ are exactly $\varphi_{*} \mathcal{D}_{k}$. The two first relations in (II.8) are obvious from (II.4) and (II.7) and the fourth one is a consequence of the third one because $\mathcal{D}_{k} \subset\left(\mathcal{D}_{k}^{\perp}\right)^{\perp}$, with an equality at nonsingular points. Let us prove the first one by induction. It is obvious for $k \leq 1$. Let us suppose that it is true for $k \geq 1$. From the fact that if $\langle\omega, X\rangle=0$ then $\left\langle L_{F} \omega, X\right\rangle=-\langle\omega,[F, X]\rangle$, we have :

$$
\begin{aligned}
\omega \in \mathcal{H}_{k+1} & \Leftrightarrow \omega \in \mathcal{H}_{k} \text { and } L_{\varphi} \omega \in \mathcal{H}_{k} \\
& \Leftrightarrow \forall X \in \mathcal{D}_{k},\langle\omega, X\rangle=\left\langle L_{F} \omega, X\right\rangle=0 \\
& \Leftrightarrow \forall X \in \mathcal{D}_{k}, \quad\langle\omega, X\rangle=\langle\omega,[F, X]\rangle=0 \quad \Leftrightarrow \omega \in \mathcal{D}_{k+1}^{\perp} .
\end{aligned}
$$

We shall now relate this construction to accessibility. The following Lie algebra is defined in [38], and often called the strong accessibility Lie algebra : this Lie algebra of vector fields on $\mathbb{R}^{n}$ is the Lie ideal generated by all the vector fields $f(u,)-.f(v,$.$) for all possible$ 
values of $u$ and $v$ in the Lie algebra generated by the vector fields $f(u,$.$) for all possible$ values of $u$. The main result on strong accessibility in [38] (see the definition there) is that it is equivalent to the strong accessibility Lie algebra having rank $n$. In [9], the strong jet accessibility Lie algebra is defined; it differs from the strong accessibility Lie algebra in that the differences $f(u,)-.f(v,$.$) are replaced by derivatives of all orders with respect to$ all the components of $u$. It is easy to see (this is actually its definition in [9]) that it is the Lie algebra generated by all the vector fields

$$
\operatorname{ad}_{f(., u)}^{j} g_{u}^{K}, \quad j \in \mathbb{N}, \quad K=\left(k_{1}, \ldots, k_{m}\right) \in \mathbb{N}^{m}, g_{u}^{K}=\frac{\partial^{k_{1}+\ldots+k_{m}} f}{\partial u_{1}^{k_{1}} \ldots \partial u_{m}^{k_{m}}} .
$$

It a priori depends on $u$. In the analytic case, it does not depend on $u$ and is equal, for all value of $u$, to the strong accessibility Lie algebra. Of course, in the general (smooth) case, full rank for this Lie algebra is sufficient, but not necessary, for strong accessibility. A vector field on $\mathbb{R}^{n}$ depending on $u$, like these defined in (II.9) and all their iterated Lie brackets, clearly defines a vector field on $\mathcal{M}_{\infty}^{m, n}$ (which belongs to $\operatorname{Span}\left\{\frac{\partial}{\partial x}\right\}$ and commutes with all the $\frac{\partial}{\partial u_{k}^{(j)}}$ for $j \geq 1$ but not a priori with the $\frac{\partial}{\partial u_{k}}$ 's). Here, we call $\widehat{\mathcal{L}}$ the Lie algebra composed of the vector fields on $\mathcal{M}_{\infty}^{m, n}$ associated to these in the strong jet accessibility Lie algebra as defined by (II.9) (or in [9]), and we define $\mathcal{L}$ by

$$
\mathcal{L}=\widehat{\mathcal{L}} \oplus \mathcal{D}_{1}=\widehat{\mathcal{L}} \oplus \operatorname{Span}\left\{\frac{\partial}{\partial u}, \frac{\partial}{\partial \dot{u}}, \frac{\partial}{\partial \ddot{u}}, \frac{\partial}{\partial u^{(3)}}, \ldots\right\} .
$$

$\mathcal{L}$ is obviously a Lie algebra because $\left[\frac{\partial}{\partial u_{k}}, \widehat{\mathcal{L}}\right] \subset \widehat{\mathcal{L}}$ and $\left[\frac{\partial}{\partial u_{k}^{(j)}}, \widehat{\mathcal{L}}\right]=\{0\}$ for $j \geq 1$. The phrase "strong jet accessibility Lie algebra" will further refer to $\mathcal{L}$ rather than to a Lie algebra of vector fields on $\mathbb{R}^{n}$, and $\widehat{\mathcal{L}}$ is its $\frac{\partial}{\partial x}$-component. We have :

Theorem II.1 For any open subset $U$ of $\mathcal{M}_{\infty}^{m, n}$,

1. $\left.\mathcal{L}\right|_{U}$ (restriction to $U$ of the strong jet accessibility Lie Algebra) is the Lie Algebra generated by (i.e. the involutive closure of) $\left.\mathcal{D}_{\infty}\right|_{U}$ (the restriction of $\mathcal{D}_{\infty}$ to $U$ ).

2. If the $\mathcal{C}^{\infty}$-module $\left.\widehat{\mathcal{D}}_{\infty}\right|_{U}$ is finitely generated, then it is a Lie algebra, and so is $\left.\mathcal{D}_{\infty}\right|_{U}$, and hence :

$$
\left.\mathcal{D}_{\infty}\right|_{U}=\left.\mathcal{L}\right|_{U} \quad \text { i.e. }\left.\quad \widehat{\mathcal{D}}_{\infty}\right|_{U}=\left.\widehat{\mathcal{L}}\right|_{U} .
$$

Proof : Point 1 is straightforward from (II.9) and (II.4). We only have to prove that if $U$ is such that $\left.\widehat{\mathcal{D}}_{\infty}\right|_{U}$ is finitely generated, then $\left.\mathcal{D}_{\infty}\right|_{U}$ is a Lie algebra ; for this, we shall prove that the module of vector fields

$$
\mathcal{M}=\left\{\left.X \in \mathcal{D}_{\infty}\right|_{U},\left.\left[X,\left.\mathcal{D}_{\infty}\right|_{U}\right] \subset \mathcal{D}_{\infty}\right|_{U}\right\}
$$

is equal to $\left.\mathcal{D}_{\infty}\right|_{U}$. By assumption, $\left.\mathcal{D}_{\infty}\right|_{U}$ is generated by the vector fields $\frac{\partial}{\partial u_{k}^{(j)}}, 1 \leq k \leq m$, $j \geq 0$, plus a finite number of vector fields of $\operatorname{Span}\{\mathrm{d} x\}$ whose expressions involve only a finite number, say $J$, of time-derivatives of $u ;\left.\mathcal{D}_{\infty}\right|_{U}$ is therefore invariant by Lie bracket by the vector fields $\frac{\partial}{\partial u_{k}^{(j)}}$ for $j \geq J$, which span $\mathcal{D}_{-(J-1)} \cdot \mathcal{M}$ therefore contains $\mathcal{D}_{-(J-1)}$; 
furthermore, it is a submodule of $\left.\mathcal{D}_{\infty}\right|_{U}$, invariant by $F$ from Jacobi identity. Since it is clear that, for all $k$, and in particular $k=-(J-1),\left.\mathcal{D}_{\infty}\right|_{U}$ is the smallest module of vector fields which contains $\mathcal{D}_{k}$ and is invariant by Lie brackets by $F, \mathcal{M}=\left.\mathcal{D}_{\infty}\right|_{U}$.

For further considerations, we will avoid "singular" points in the sense of the following definition where $\mathcal{H}_{k}+\dot{\mathcal{H}}_{k}$ stands for the module over smooth functions spanned by all the forms $\omega$ and $\dot{\omega}$ with $\omega \in \mathcal{H}_{k}$. "Nonsingular" was defined in paragraph 2.9.

Definition II.2 A point $\mathcal{X} \in \mathcal{M}_{\infty}^{m, n}$ is called a Brunovský-regular point for system $F$ if and only if one of the two following (equivalent) conditions is satisfied:

(i) All the modules $\widehat{\mathcal{D}}_{k}(k \geq 2)$ are nonsingular at $\mathcal{X}$.

(ii) All the modules $\mathcal{H}_{k}+\dot{\mathcal{H}}_{k}(k \geq 2)$ are nonsingular at $\mathcal{X}$.

These properties are true for all $k \geq 0$ if and only if they are true for $k=2, \ldots, n+1$. We call $\boldsymbol{\rho}_{\boldsymbol{k}}$ the locally constant rank of $\mathcal{H}_{k}$. Around a Brunovsky-regular point, there exists an integer $\boldsymbol{k}^{*}$ such that, for all $k \leq k^{*}, \rho_{k+1} \leq \rho_{k}-1$ and $\mathcal{H}_{k}=\mathcal{H}_{k+1}=\mathcal{H}_{\infty}$ for $k>k^{*}$.

Proof of $\boldsymbol{i} \Leftrightarrow \boldsymbol{i i}$ : Suppose that all the $\widehat{\mathcal{D}}_{k}$ 's, and thus all the $\mathcal{H}_{k}$ 's, are nonsingular at $\mathcal{X}$. For a certain $k$, let $\left\{\eta_{1}, \ldots, \eta_{p+q}\right\}$ be a basis of $\mathcal{H}_{k}$ with $\left\{\eta_{1}, \ldots, \eta_{p}\right\}$ a basis of $\mathcal{H}_{k+1}$. The forms $\eta_{1}, \ldots, \eta_{p+q}, \dot{\eta}_{p+1}, \ldots, \dot{\eta}_{p+q}$ span $\mathcal{H}_{k}+\dot{\mathcal{H}}_{k}$. On the other hand, if a linear combination $\sum_{i=1}^{p+q} \mu_{i} \eta_{i}+\sum_{i=1}^{q} \lambda_{i} \dot{\eta}_{p+i}$ vanishes at $\mathcal{X}$ then, for all vector field $X \in \mathcal{D}_{k},\left\langle\sum_{i=1}^{q} \lambda_{i} \dot{\eta}_{p+i}, X\right\rangle$, which is equal to $\left\langle\sum_{i=1}^{q} \lambda_{i} \eta_{p+i},[F, X]\right\rangle$, vanishes at $\mathcal{X}$, hence $\left\langle\sum_{i=1}^{q} \lambda_{i} \eta_{p+i}, Y\right\rangle(\mathcal{X})=0$ for all $Y \in \mathcal{D}_{k+1}$; since $\left\{\eta_{1}(\mathcal{X}), \ldots, \eta_{p}(\mathcal{X})\right\}$ is a basis of the annihilator of $\mathcal{D}_{k+1}(\mathcal{X})$ and $\left\{\eta_{1}(\mathcal{X}), \ldots, \eta_{p+q}(\mathcal{X})\right\}$ are independent, all the $\lambda_{i}$ 's vanish at $\mathcal{X}$; hence $\sum_{i=1}^{p+q} \mu_{i} \eta_{i}$ vanishes at $\mathcal{X}$, hence all the $\mu_{i}$ 's also vanish at $\mathcal{X}$. Hence $\left\{\eta_{1}(\mathcal{X}), \ldots, \eta_{p+q}(\mathcal{X}), \dot{\eta}_{p+1}(\mathcal{X}), \ldots, \dot{\eta}_{p+q}(\mathcal{X})\right\}$ is a basis of $\mathcal{H}_{k}(\mathcal{X})+\dot{\mathcal{H}}_{k}(\mathcal{X})$ and $\mathcal{H}_{k}+\dot{\mathcal{H}}_{k}$ is nonsingular at $\mathcal{X}$.

Conversely suppose that all the modules $\mathcal{H}_{k}+\dot{\mathcal{H}}_{k}$ are nonsingular at $\mathcal{X}$. Let $\mathcal{C}_{k}=$ $\left\{X \in \mathcal{D}_{k},[F, X] \in \mathcal{D}_{k}\right\}$ and $\widehat{\mathcal{C}_{k}}=\mathcal{C}_{k} \cap \operatorname{Span}\left\{\frac{\partial}{\partial x}, \frac{\partial}{\partial u}\right\}$. Clearly, $\mathcal{C}_{k}=\widehat{\mathcal{C}_{k}} \oplus \mathcal{D}_{0}$. Arguments similar to these of the end of the proof of Proposition II.1 show that $\left(\mathcal{H}_{k}+\dot{\mathcal{H}}_{k}\right)^{\perp}=\mathcal{C}_{k}$ (equality between modules). All the $\widehat{\mathcal{C}_{k}}$ 's are therefore nonsingular at $\mathcal{X}$. Let us prove by induction that all the modules $\widehat{\mathcal{D}}_{k}$ are nonsingular too. This is true for $k=1\left(\widehat{\mathcal{D}}_{1}=\right.$ $\{0\})$. Suppose that it is true for $k \geq 1$, and let $\left\{\ldots, \frac{\partial}{\partial \ddot{u}}, \frac{\partial}{\partial \dot{u}}, X_{1}, \ldots, X_{p+q}\right\}$ be a basis of $\mathcal{D}_{k}$ with $\left\{\ldots, \frac{\partial}{\partial \ddot{u}}, \frac{\partial}{\partial \dot{u}}, X_{1}, \ldots, X_{p}\right\}$ a basis of $\mathcal{C}_{k}$. Then the same arguments as in the first part of this proof show that $\left\{X_{1}(\mathcal{X}), \ldots, X_{p+q}(\mathcal{X}),\left[F, X_{p+1}\right](\mathcal{X}), \ldots,\left[F, X_{p+q}\right](\mathcal{X})\right\}$ is a basis of $\operatorname{Span}\left\{\frac{\partial}{\partial u}\right\} \oplus \mathcal{D}_{k+1}(\mathcal{X})$ and $\widehat{\mathcal{D}}_{k+1}$ is nonsingular at $\mathcal{X}$.

Theorem II.2 (Infinitesimal Brunovský form) Around a Brunovský-regular point there exists $\rho_{\infty}$ functions of $x$ only $\chi_{1}, \ldots, \chi_{\rho_{\infty}}$, and $m 1$-forms $\omega_{1}, \ldots, \omega_{m}$, and $m$ non-negative integers $r_{1} \ldots, r_{m}$ such that

$$
\begin{aligned}
& \left\{\mathrm{d} \chi_{1}, \ldots, \mathrm{d} \chi_{\rho_{\infty}}\right\} \text { is a basis of } \mathcal{H}_{\infty}=\mathcal{H}_{l}, \text { for } l \geq k^{*}+1 \\
& \left\{\mathrm{~d} \chi_{1}, \ldots, \mathrm{d} \chi_{\rho_{\infty}}\right\} \cup\left\{\omega_{k}^{(j)}, r_{k} \geq l, 0 \leq j \leq r_{k}-l\right\} \text { is a basis of } \mathcal{H}_{l} \text {, for all } l \leq k k^{*}
\end{aligned}
$$

Furthermore all the $\omega_{k}$ 's are in $\mathcal{H}_{1}=\operatorname{Span}\{\mathrm{d} x\}-$ i.e. $r_{k} \geq 1$ for all $k$ - if and only if, at the point $(x, u)$ under consideration,

$$
\operatorname{rank}_{\mathbb{R}}\left\{\frac{\partial f}{\partial u_{1}}(x, u), \ldots, \frac{\partial f}{\partial u_{m}}(x, u)\right\}=m .
$$


At a Brunovský-regular point, $\mathcal{D}_{\infty}$ is equal to $\mathcal{D}_{n+1}$ and is hence nonsingular and hence locally finitely generated. Hence strong accessibility implies, from Theorem II.1, that $\rho_{\infty}=0$. In that case and if (II.14) is met, (II.13) implies

$$
\begin{aligned}
& \left\{\omega_{k}^{(j)}, 0 \leq k \leq m, 0 \leq j \leq r_{k}-1\right\} \text { is a basis of } \mathcal{H}_{1}=\operatorname{Span}\{\mathrm{d} x\} \\
& \left\{\omega_{k}^{(j)}, 0 \leq k \leq m, 0 \leq j \leq r_{k}\right\} \text { is a basis of } \mathcal{H}_{0}=\operatorname{Span}\{\mathrm{d} x, \mathrm{~d} u\}
\end{aligned}
$$

Hence, with $\omega_{k, j}=\omega_{k}^{(j)}$, and with the $a_{i, j}$ 's and $b_{i, j}$ 's some functions such that the matrix $\left[b_{i, j}\right]_{i, j}$ is invertible at $\mathcal{X}$

$$
\left.\begin{array}{rl}
\dot{\chi}_{1}= & \gamma_{1}\left(\chi_{1}, \ldots, \chi_{\rho_{\infty}}\right) \\
\vdots & \\
\dot{\chi}_{\rho_{\infty}}= & \gamma_{\rho_{\infty}}\left(\chi_{1}, \ldots, \chi_{\rho_{\infty}}\right) \\
\dot{\omega}_{i, 1} & =\omega_{i, 2} \\
\dot{\omega}_{i, 2} & =\omega_{i, 3} \\
& \vdots \\
\dot{\omega}_{i, r_{i}-1} & =\omega_{i, r_{1}} \\
\dot{\omega}_{i, r_{1}} & =\sum_{j=1}^{n} a_{i, j} \mathrm{~d} x_{j}+\sum_{j=1}^{m} b_{i, j} \mathrm{~d} u_{j}
\end{array}\right\} 1 \leq i \leq m .
$$

We call this "infinitesimal Brunovský form" because it looks like the canonical Brunovský form [5] for linear system; it is not a "canonical form" for any equivalence relation : the data of the forms $\omega_{1}, \ldots, \omega_{m}$ and of (II.16) does not give a unique system.

Proof : The proof goes along the lines of [2] or [33]. Since we are at a Brunovský-regular point, $\mathcal{H}_{\infty}$ is nonsingular and locally spanned by exactly $\rho_{\infty}$ forms. These forms depend on a finite number of variables $x, u, \ldots, u^{(K)}$. One may then project these forms, and hence $\mathcal{H}_{\infty}$, on the finite dimensional manifold $\mathcal{M}_{K}^{m, n}$ (see Section I.3.2) and use the finite dimensional Frobenius theorem : from Theorem II.1, $\mathcal{H}_{\infty}$ is completely integrable and therefore is spanned by $\rho_{\infty}$ exact forms $\mathrm{d} \chi_{1} \ldots \mathrm{d} \chi_{\rho_{\infty}}$ with $\chi_{1} \ldots \chi_{\rho_{\infty}}$ some functions, which depend only on $x$ because $\mathrm{d} \chi_{i} \in \mathcal{D}_{\infty} \subset \mathcal{D}_{1}$. Then the forms $\omega_{k}$ may be constructed recursively such that (II.13) holds :

- it holds for $l \geq k^{*}+1$ provided all the $r_{k}$ 's are no larger than $k^{*}$ (it will be the case).

- chose $\omega_{1}, \ldots, \omega_{\rho_{k^{*}}}$ so that $\left\{\mathrm{d} \chi_{1}, \ldots, \mathrm{d} \chi_{\rho_{\infty}}, \omega_{1}, \ldots, \omega_{\rho_{k^{*}}}\right\}$ is a basis of $\mathcal{H}_{k^{*}}$, and set $r_{1}=\ldots=$ $r_{\rho_{k^{*}}}=k^{*}$, (II.13) is then satisfied for $l \geq k^{*}$ provided all the remaining $r_{k}$ 's are no larger than $k^{*}-1$ (it will be the case).

- Induction on $\ell$, downward from $\ell=k^{*}$ to $\ell=0$ : for $0 \leq \ell \leq k^{*}-1$, let us suppose that (II.13) is true for $l \geq \ell+1$ (assuming that all the $r_{k}$ 's corresponding to $\omega_{k}$ 's which have not yet been built are no larger than $\ell$ ), and build some $\omega_{k}$ 's with $r_{k}=\ell$ so that (II.13) is true for $l \geq \ell$. It is not difficult to prove (see [2, proof of Th. 3.5], really similar because by assumption $\mathcal{H}_{\ell+1}+\dot{\mathcal{H}}_{\ell+1}$ is nonsingular here) that $\left\{\mathrm{d} \chi_{1}, \ldots, \mathrm{d} \chi_{\rho_{\infty}}\right\} \cup\left\{\omega_{k}^{(j)}, r_{k} \geq \ell+1,0 \leq j \leq r_{k}-\ell\right\}$ is a set of linearly independent elements of $\mathcal{H}_{\ell}$, actually a basis of $\mathcal{H}_{\ell+1}+\dot{\mathcal{H}}_{\ell+1} \subset \mathcal{H}_{\ell}$. Add, if they do not form a basis of $\mathcal{H}_{\ell}$, some new $\omega_{k}$ 's with the corresponding $r_{k}$ 's equal to $\ell$.

After $l=0$, no new $\omega_{k}$ 's are needed because if there is a certain number of $\omega_{k}$ 's such that (II.13) holds for $l=0$ (we have not yet proved there are exactly $m$ of them), then $\mathrm{d} u_{1}, \ldots, \mathrm{d} u_{m}$ are linear combinations of the $\mathrm{d} \chi_{i}$ 's and the $\omega_{k}^{(j)}$ 's for $r_{k} \geq 0$ and $0 \leq j \leq r_{k}$, which immediately implies that, for $q>0, \mathrm{~d} u_{1}^{(q)}, \ldots, \mathrm{d} u_{m}^{(q)}$ are linear combinations of the 
$\mathrm{d} \chi_{i}$ 's and the $\omega_{k}^{(j)}$ 's for $r_{k} \geq 0$ and $0 \leq j \leq r_{k}+q$, i.e. (II.13) is met for $l=-q<0$ without any additional $\omega_{k}$ 's; this ends the construction of the $\omega_{k}$ 's and proves $r_{k} \geq 0$ for all $k$. There are exactly $m \omega_{k}$ 's because an obvious consequence of (II.13) is that $\rho_{l}-\rho_{l+1}$ is equal to the number of $r_{k}$ 's larger or equal to $l$; in particular, since $\rho_{l}-\rho_{l+1}=m$ for $l \leq 0$ (see (II.7)), the total number of $\omega_{k}$ 's is $m$. To prove the very last part of the theorem, one therefore has to prove that $\rho_{1}-\rho_{2}=m$ if and only if (II.14) holds, which is obvious because, from (II.4), $\mathcal{D}_{2}=\mathcal{D}_{1} \oplus \operatorname{Span}\left\{\frac{\partial f}{\partial u_{1}}, \ldots, \frac{\partial f}{\partial u_{m}}\right\}$ and because of Brunovský-regularity.

The reason for defining this "Brunovský form" in $[2,33]$ was to suggest a way to look for "linearizing outputs" (see theorem II.3 below for definition and comments).

Definition I.4 introduces, as in [2,33], the notion of a linearizing Pfaffian system. Recall that one should not be mislead by the terminology : a linearizing Pfaffian system, contrary to a linearizing output, does not linearize anything unless it has more properties (integrability, see Theorem II.3). An an immediate consequence of Theorems II.1 and II.2 is :

Corrolary II.2 If a system $F$ is locally strongly accessible around a point $\mathcal{X}$, which is Brunovský-regular for $F$, then $F$, admits, locally around $\mathcal{X}$, a linearizing Pfaffian system $\left(\omega_{1}, \ldots, \omega_{m}\right)$. A possible choice is the forms $\omega_{1}, \ldots, \omega_{m}$ constructed in Theorem II.2. If (II.14) holds, $\omega_{1}, \ldots, \omega_{m}$ are in $\mathcal{H}_{1}=\operatorname{Span}\{\mathrm{d} x\}$.

\section{Comments on this "Brunovský form"}

Let us indicate the similarity between the content of this section and the algebraic framework for "time-varying" linear systems developed in $[13,14]$ for example.

For $U$ an open subset of $\mathcal{M}_{\infty}^{m, n}$, let $\mathcal{C}^{\infty}(U)\left[L_{F}\right]$ be the algebra of differential operators which are polynomials in the Lie derivative with respect to $F$ with coefficients in $\mathcal{C}^{\infty}(U)$. This is a non-commutative algebra since $\left(a L_{F}\right)\left(b L_{F}\right)=a b L_{F}^{2}+a\left(L_{F} b\right) L_{F}$. It plays the same role as the non-commutative ring $k\left[\frac{\mathrm{d}}{\mathrm{dt}}\right]$ ( $k$ is a differential field) introduced in [13] to define linear time-varying systems : a linear system is a module over this ring and it is controllable if and only if it is a free $k\left[\frac{\mathrm{d}}{\mathrm{dt}}\right]$-module (which is also a $k$ vector space).

In the nonlinear case, in $[13,15]$ a system is represented by a differential field $k$ and, via Kähler differentials, one may define the linearized system as a $k\left[\frac{\mathrm{d}}{\mathrm{dt}}\right]$-module, whose equivalent here is the $\mathcal{C}^{\infty}(U)\left[L_{F}\right]$-module $\Lambda^{1}(U)$.

Relying upon results from [37, 9] which state that a nonlinear system satisfying the strong accessibility condition has a controllable linear approximation along "almost any" trajectory, a nonlinear system is said to be controllable in [15] if and only if the $k\left[\frac{\mathrm{d}}{\mathrm{dt}}\right]$-module associated to the differential field $k$ is free.

Note that the assertion " $\left(\omega_{1}, \ldots, \omega_{m}\right)$ is a linearizing Pfaffian system" (or (II.13) with $\left.\rho_{\infty}=0\right)$ is equivalent to " $\left(\omega_{1}, \ldots, \omega_{m}\right)$ is a basis of the $\mathcal{C}^{\infty}(U)\left[L_{F}\right]$-module $\Lambda^{1}(U)$ "; hence Corollary II.2 constructs a basis of this module, and hence establishes that it is free. We have proved (theorem II.1), that, at a Brunovský-regular point (and even at a point where $\widehat{\mathcal{D}}_{\infty}$ is locally finitely generated), the strong accessibility rank condition implies that the module is free, or that the linearized system is controllable in the sense of $[13,15]$. This is not exactly a consequence of $[37,9]$. Technically, the result is contained in the fact that $\mathcal{D}_{\infty}$ is (around a regular point) closed under Lie bracket, which may be interpreted as : the torsion submodule of the $\mathcal{C}^{\infty}(U)\left[L_{F}\right]$-module $\Lambda^{1}(U)$ is "integrable". 
An algebraic construction of the "canonical Brunovský form" (or of a basis of the module) for controllable time-varying linear systems, based on some filtrations, is proposed in [14]. The sequence of the $\mathcal{H}_{k}$ 's is a filtration of $\Lambda^{1}(U)$. It does not coincide with these introduced in [15], but might certainly be interpreted in the same terms. The "well-formedness" assumption in [15] corresponds to (II.14) at the end of Theorem II.2.

\section{II.4 Dynamic linearization as an integrability problem}

Dynamic linearizability from Definition II.1 is actually linearizability by endogenous dynamic feedback as defined in $[26,16,17]$. It is proved there that this is equivalent to flatness, i.e. to existence of linearizing outputs or flat outputs. In the present framework, these are defined below. They are given an interpretation in terms of dynamic decoupling and structure at infinity in [2] and in [26], and they are defined as the free generators of the differential algebra $\mathcal{C}^{\infty}\left(\mathcal{M}_{\infty}^{m, n}\right)$ in $[23,24]$.

The following is an immediate consequence of Theorems I.4 and I.5 :

Theorem II.3 Let $\mathcal{X}$ be a point of $\mathcal{M}_{\infty}^{m, n}$. The following assertions are equivalent:

1. The system $F$ is locally dynamic linearizable at point $\mathcal{X}$.

2. There exist $m$ smooth functions $h_{1}, \ldots, h_{m}$ from a neighborhood of $\mathcal{X}$ in $\mathcal{M}_{\infty}^{m, n}$ to $\mathbb{R}$ such that $\left(L_{F}^{j} h_{k}\right)_{1 \leq k \leq m, 0 \leq j}$ is a local system of coordinates at $\mathcal{X}$. Such $m$ functions are called linearizing outputs (or simply one linearizing output) $[16,17,26]$.

3. $F$ admits, on a neighborhood of $\mathcal{X}$, a linearizing Pfaffian system $\left(\eta_{1}, \ldots, \eta_{m}\right)$ which is completely integrable, i.e. such that $\mathrm{d} \eta_{k} \wedge \eta_{1} \wedge \ldots \wedge \eta_{m}=0, k=1 \ldots m$.

We saw in the previous section that all strongly accessible systems admit, at Brunovskýregular points, a linearizing Pfaffian system, which, of course, may not be integrable. We therefore have to investigate what all linearizing Pfaffian systems are, and we may say that a system is dynamic linearizable if and only if there exists one among all these which is integrable.

For an open subset $U$ of $\mathcal{M}_{\infty}^{m, n}$, let $\mathcal{A}(U)$ be the algebra of $m \times m$ matrices with entries in the algebra of differential operators $\mathcal{C}^{\infty}(U)\left[L_{F}\right]$ :

$$
\mathcal{A}(U) \triangleq \mathcal{M}_{m \times m}\left(\mathcal{C}^{\infty}(U)\left[L_{F}\right]\right)
$$

A matrix in $\mathcal{A}(U)$ defines an operator on $m$-uples of 1 -forms in a straightforward manner, and we have :

Proposition II.3 Let $\left(\omega_{1}, \ldots, \omega_{m}\right)$ be a linearizing Pfaffian system and let $\eta_{1}, \ldots, \eta_{m}$ be $m$ 1 -forms defined on an open set $U$ of $\mathcal{M}_{\infty}^{m, n} .\left(\eta_{1}, \ldots, \eta_{m}\right)$ is a linearizing Pfaffian system if and only if there exists $P\left(L_{F}\right)$ in $\mathcal{A}(U)$ which is invertible in $\mathcal{A}(U)$ and is such that

$$
\left(\begin{array}{c}
\eta_{1} \\
\vdots \\
\eta_{m}
\end{array}\right)=P\left(L_{F}\right)\left(\begin{array}{c}
\omega_{1} \\
\vdots \\
\omega_{m}
\end{array}\right)
$$


Proof : There always exists $P\left(L_{F}\right) \in \mathcal{A}(U)$ such that (II.18) holds because $\left(\omega_{1}, \ldots, \omega_{m}\right)$ is a linearizing Pfaffian system. If $\left(\eta_{1}, \ldots, \eta_{m}\right)$ is also a linearizing Pfaffian system, there exists $Q\left(L_{F}\right) \in \mathcal{A}(U)$ such that

$$
\left(\begin{array}{c}
\omega_{1} \\
\vdots \\
\omega_{m}
\end{array}\right)=Q\left(L_{F}\right)\left(\begin{array}{c}
\eta_{1} \\
\vdots \\
\eta_{m}
\end{array}\right) .
$$

Hence $Q\left(L_{F}\right) P\left(L_{F}\right)$ and $P\left(L_{F}\right) Q\left(L_{F}\right)$ transform respectively $\left(\omega_{1}, \ldots, \omega_{m}\right)$ and $\left(\eta_{1}, \ldots, \eta_{m}\right)$ into themselves. Hence $Q\left(L_{F}\right) P\left(L_{F}\right)=P\left(L_{F}\right) Q\left(L_{F}\right)=I$ because the forms $\omega_{k}^{(j)}$ (resp. $\left.\eta_{k}^{(j)}\right)$, $1 \leq k \leq m, j \geq 0$, are linearly independent. Conversely, it is obvious that (II.18) with $P\left(L_{F}\right)$ invertible implies that $\left(\eta_{k}^{(j)}\right)_{1 \leq k \leq m, j \geq 0}$ is a basis of the $\mathcal{C}^{\infty}(U)$-module $\Lambda^{1}(U)$.

A straightforward consequence of Theorem II.2 and Proposition II.3 is :

Theorem II.4 Let $\mathcal{X} \in \mathcal{M}_{\infty}^{m, n}$ be a Brunovský-regular point for system $F$, and let $\omega_{1}, \ldots, \omega_{m}$ be the 1-forms constructed in Theorem II.2, defined on a certain neighborhood $U$ of $\mathcal{X}$. System $F$ is locally dynamic linearizable at point $\mathcal{X}$ if and only if there exists an invertible matrix $P\left(L_{F}\right) \in \mathcal{A}(U)$ such that

$$
\left(\begin{array}{c}
\bar{\omega}_{1} \\
\vdots \\
\bar{\omega}_{m}
\end{array}\right)=P\left(L_{F}\right)\left(\begin{array}{c}
\omega_{1} \\
\vdots \\
\omega_{m}
\end{array}\right)
$$

is a locally completely integrable Pfaffian system, i.e. $\mathrm{d} \bar{\omega}_{k} \wedge \bar{\omega}_{1} \wedge \ldots \wedge \bar{\omega}_{m}=0$ for $k=1, \ldots, m$.

Of course, this is not per se a solution to the dynamic feedback linearization problem; it is rather a convenient way to pose the problem of deciding whether or not linearizing outputs exist. The main difficulty comes from the fact that the degree of $P$ may be arbitrarily large because the linearizing outputs may depend on an arbitrary number of time-derivatives of $u$. Let us make this number artificially finite :

Definition II.4 System $F$ is said to be $\left(\boldsymbol{x}, \boldsymbol{u}, \ldots, \boldsymbol{u}^{(\boldsymbol{K})}\right)$-linearizable (for $K=-1$, this reads $x$-linearizable) at point $\mathcal{X}$ if and only if there exists some linearizing outputs function of $\left(x, u, \ldots, u^{(K)}\right)$ only (on $x$ only for $K=-1$ ).

Of course, a system is dynamic feedback linearizable (in the sense of Definition II.1, i.e. linearizable by endogenous dynamic feedback according to $[16,17,26]$, or dynamic linearizable according to $[23,24])$ if and only if it is $\left(x, u, \ldots, u^{(K)}\right)$-linearizable for a certain $K$. We have the following theorem which precises Theorem II.4.

Theorem II.5 Let $\mathcal{X} \in \mathcal{M}_{\infty}^{m, n}$ be a Brunovský-regular point for system $F$, and let $\omega_{1}, \ldots$, $\omega_{m}$, and $r_{1}, \ldots, r_{m}$ be, respectively, the 1-forms and integers constructed in Theorem II.2. System $F$ is $\left(x, u, \ldots, u^{(K)}\right)$-linearizable at point $\mathcal{X}$ if and only if there exists an invertible matrix $P\left(L_{F}\right) \in \mathcal{A}(U)$ satisfying the conditions of Theorem II.4 and such that the degree of the entries of the $k$-th column is at most $K+r_{k}$.

Proof : The condition is necessary for $\left(x, u, \ldots, u^{(K)}\right)$-linearizability because if $h_{1}, \ldots, h_{m}$ are some linearizing outputs function of $x, u, \ldots, u^{(K)}$ only, (II.19) holds with $\bar{\omega}_{k}=\mathrm{d} \varphi_{k}$ and, from (II.13), the columns of $P$ have to satisfy the degree inequalities. Conversely, 
suppose that (II.19) holds with the degree of the $k$ th column of $P$ being at most $K+r_{k}$ and the system $\left(\bar{\omega}_{1}, \ldots, \bar{\omega}_{m}\right)$ completely integrable, then $\left(\bar{\omega}_{1}, \ldots, \bar{\omega}_{m}\right)$ is spanned by some exact forms $\left(\mathrm{d} h_{1}, \ldots, \mathrm{d} h_{m}\right)$; the functions $h_{k}$ are linearizing outputs; the degree inequalities imply that all the $\bar{\omega}_{k}$ 's are in $\mathcal{H}_{-K}=\operatorname{Span}\left\{\mathrm{d} x, \mathrm{~d} u, \ldots, \mathrm{d} u^{(K)}\right\}$, and hence that the $h_{k}$ 's are functions of $x, u, \ldots, u^{(u)}$ only.

One of the reasons why our results provide a rather convenient framework is that, outside some singular points, it is not difficult to describe invertible matrices of a prescribed degree. As noticed in $[14,15,22]$, the polynomial ring $\mathcal{C}^{\infty}(U)\left[L_{F}\right]$ enjoys many interesting properties. Namely, it is possible to perform right and left Euclidean division by a polynomial whose leading coefficient does not vanish. It is well known (see for example [41]) that, in the constant coefficient case, all invertible polynomial matrices are finite products of "elementary matrices", i.e. either diagonal invertible matrices or permutation matrices or matrices whose diagonal entries are all equal to 1 while only one of the non-diagonal entries is nonzero, and it is an arbitrary polynomial. Since the tool to get such a decomposition is only Euclidean division, this remains true in the case of coefficients in $\mathcal{C}^{\infty}(U)$ as long as one does not have to perform Euclidean division by a polynomial whose leading coefficient vanishes. This does not happen often, although it is not very easy in general to say which singularities the original matrix should not have for this not to happen; in the meromorphic case $([2,33])$, this never happens since the coefficient of the polynomials then belong to a field and are therefore invertible, even if they "vanish" at a point, if they are not zero. Now, if one bounds a priori the degree of the columns of $P$ (say one wishes to decide whether $\left(x, u, \ldots, u^{(K)}\right.$ )linearizability holds), then all invertible matrices satisfying these bounds may be sorted into a finite number of types of finite products of elementary matrices, each type involving a finite number of functions. In each case,

$$
\mathrm{d}\left(P\left(L_{F}\right)\left(\begin{array}{c}
\omega_{1} \\
\vdots \\
\omega_{m}
\end{array}\right)\right)=0
$$

(with d acting on each entry) is a set of partial differential equations in these functions. The solubility of these PDE's is equivalent to the existence of a system of linearizing outputs depending only on a fixed finite number of time-derivatives of $u$.

\section{II.5 Conclusion}

We have developed a framework for looking for linearizing outputs which gives a convenient way for writing down a system of equations whose solubility is equivalent to the existence of a system of linearizing outputs. Some work has already been done in the direction of characterizing the cases where linearizing outputs exist. These results give either sufficient conditions or necessary and sufficient conditions for existence of linearizing outputs for some particular cases. For example, $\left(x, u, \ldots, u^{(K)}\right)$-linearizability (in most cases, $K=-1$ ) or a prescribed "structure at infinity" (see $[27,28,29]$ ). A criterion for existence of a matrix $P$ of degree zero for general two-inputs systems is given in [33]. The "sufficiency" part of the result contained in [29] is re-derived in [3] in a way that simplifies, to our opinion, the argument partly due to E. Cartan. Finally, a characterization of $(x, u)$-linearizability for affine systems 
with 4 states and 2 inputs is given in [32]. These last results seem to demonstrate that "infinitesimal Brunovský form" is a convenient way to tackle the problem of looking for linearizing outputs.

\section{Acknowledgments}

The authors wish to acknowledge some discussions with Michel Fliess on the relation between the linear Brunovský canonical form for time-varying linear systems and the "infinitesimal Brunovský form" described here, or in their original work [33]. They also thank him for communicating the preprint [14]. 


\section{Bibliography}

[1] R.L. Anderson, N.H. Ibragimov, Lie-Bäcklund Transformations in Applications. SIAM studies in Applied Mathematics, SIAM, Philadelphia, 1979.

[2] ( $\left.{ }^{1}\right)$ E. Aranda-Bricaire, C. H. Moog, J.-B. Pomet, "A linear algebraic framework for dynamic feedback linearization". IEEE Trans. Autom. Contr., to appear, 1994 or 1995.

[3] E. Aranda, C. H. Moog, J.-B. Pomet, "Feedback Linearization: A Linear Algebraic Approach". 22nd IEEE Conf. on Dec. and Cont., dec. 1993.

[4] N. Bourbaki : Eléments de Mathématique, Espaces Vectoriels Topologiques, chap. 1. Masson, Paris, 1981.

[5] P. Brunovský, "A classification of linear controllable systems". Kybernetica, 6 (1970), pp. 176-188.

[6] B. Charlet, J. Lévine, R. Marino, "On dynamic feedback linearization". Syst. Contr. Lett., 13, pp. 143-151, 1989.

[7] B. Charlet, J. Lévine, R. Marino, "Sufficient conditions for dynamic feedback linearization". SIAM J. Contr. Opt., 29, pp. 38-57, 1991

[8] G. Conte, A.-M. Perdon, C. Moog, "The Differential Field Associated to a General Analytic Nonlinear System". IEEE Trans. Autom. Contr., 38 (1993), pp. 1120-1124.

[9] J.-M. Coron, "Linearized Control Systems and Applications to Smooth Stabilization". SIAM J. Contr. Opt., 32 (1994), No 2, pp. 358-386.

[10] E. Delaleau, Sur les dérivées de l'entrée en représentation et Commande des systèmes non-linéaires. Thèse de l'Unviversité Paris XI, Orsay, 1993.

[11] M. D. Di Benedetto, J. Grizzle, C. H. Moog, "Rank Invariants of Nonlinear Systems". SIAM J. Contr. Opt., 27, pp. 658-672, 1989

[12] M. Fliess, "Décomposition en cascade des systèmes automatiques et feuilletages invariants". Bull. Soc. Math. Fr., vol. 113, pp.285-293, 1985.

[13] M. Fliess, "Automatique et corps différentiels". Forum Math., 1 (1989), pp. 227-238.

\footnotetext{
${ }^{1}$ IEEE Trans. Autom. Contr., 40 (1995), pp. 127-132
} 
[14] $\left({ }^{2}\right)$ M. Fliess, "Some remarks on the Brunovsky canonical form". Preprint, L.S.S., Ec. Sup. d'Elec., Gif-sur-Yvette, France, 1992, to appear in Kybernetika.

[15] M. Fliess, S.T. Glad, "An Algebraic Approach to Linear and Nonlinear Control". In H.L. Trentelman and J.C. Willems Eds., Essays on Control: Perspectives in the Theory and its Applications, PSCT vol. 14, Birkäuser, Boston, Basel, Berlin, 1993.

[16] M. Fliess, J. Lévine, P. Martin, P. Rouchon, "On differentially Flat Nonlinear Systems". 2nd. IFAC NOLCOS Symposium, pp. 408-412, 1992.

[17] M. Fliess, J. Lévine, P. Martin, P. Rouchon, "Sur les systèmes non linéaires différentiellement plats". C. R. Acad. Sc. Paris, 315-I, pp. 619-624, 1992.

[18] $\left(^{3}\right)$ M. Fliess, J. Lévine, P. Martin, P. Rouchon, "Linéarisation par bouclage dynamique et transformations de Lie-Bäcklund". C. R. Acad. Sc. Paris, to appear, 1993.

[19] ( $\left.{ }^{4}\right)$ M. Fliess, J. Lévine, P. Martin, P. Rouchon, "Towards a new differential geometric setting in nonlinear control". Presented at International Geometrical Colloquium, Moscow, may 1993, and to appear in the proceedings.

[20] E. Goursat, Le problème de Bäcklund. Mémorial des sciences mathématiques, fasc. VI, Gauthier-Villars, Paris, 1925.

[21] R.S. Hamilton : "The Inverse Function Theorem of Nash and Moser". Bulletin (new series) of the A.M.S., 7 (1982), pp.65-222.

[22] A. Ilchmann, I. Nürnberger, W. Schmale, "Time-varying Polynomial matrix systems". Int. J. of Control, 40 (1984), pp.329-362.

[23] B. Jakubczyk, "Remarks on Equivalence and Linearization of Nonlinear Systems". 2nd. IFAC NOLCOS Symposium, pp. 393-397, 1992.

[24] B. Jakubczyk, "Dynamic Feedback Equivalence of Nonlinear Control Systems". Preprint, 1993.

[25] I.S. Krasil'shchik, V.V. Lychagin, A.M. Vinogradov : Geometry of Jet Spaces and Nonlinear Partial Differential Equations. Adv. Stud. on Contemporary Math., vol. 1, Gordon \& Breach Sci. Publishers, 1986.

[26] P. Martin, Contribution à l'étude des systèmes non linéaires différentiellement plats. Thèse de Doctorat, Ecole des Mines de Paris, 1992.

[27] P. Martin, "A geometric sufficient conditions for flatness of systems with $m$ inputs and $m+1$ states". 22nd IEEE Conf. on Dec. and Cont., dec. 1993, pp.3431-3435.

[28] P. Martin, "A criterion for flatness with structure $\{1, \ldots, 1,2\}$ ". Preprint, 1992.

\footnotetext{
${ }^{2}$ Kybernetyka, 29 (1993), pp. 417-422.

${ }^{3}$ C. R. Acad. Sc. Paris, 317-I, pp. 981-986, 1993.

${ }^{4}$ The proceedings of that conference did unfortunately not appear. This material was subsequently published, with improvements, as "A Lie-Bäcklund approach to equivalence and flatness of nonlinear systems", IEEE Trans. Autom. Contr., 44 (1999), pp. 922-937
} 
[29] P. Martin, P. Rouchon "Systems without drift and flatness". Int. Symp. on Math. Theory on Networks and Syst., Regensburg, August 1993.

[30] P. Otterson, G. Svetlichny, "On Derivative-Dependent Deformations of Differential Maps". J. of Diff. Eq. 36 (1980), pp.270-294.

[31] F.A.E. Pirani, D.C. Robinson, W.F. Shadwick : Local jet bundle formulation of Bäcklund transformations. Mathematical Physics Studies, D. Reidel Pub. Co., London, Boston, Dordrecht, 1979.

[32] $\left({ }^{5}\right)$ J.-B. Pomet, "On dynamic feedback linearization of Four-dimensional affine control systems with two inputs". Preprint, 1993, INRIA report No 2314.

[33] J.-B. Pomet, C. H. Moog, E. Aranda, "A non-exact Brunovský form and dynamic feedback linearization". Proc. 31st. IEEE Conf. Dec. Cont., pp. 2012-2017, 1992.

[34] J.-F. Pommaret, "Géométrie différentielle algébrique et théorie du contrôle". C. R. Acad. Sc. Paris, t. 302, série I, No15, pp. 547-550, 1986.

[35] D.J. Saunders, The Geometry of Jet Bundles London Math. Soc. Lect. Notes vol. 142, Cambridge University Press, Cambridge, New-York, Melbourne, 1989.

[36] W.F. Shadwick : "Absolute Equivalence and Dynamic Feedback Linearization". Syst. Contr. Lett., 15 (1990), pp. 35-39.

[37] E.D. Sontag, "Finite-dimensional open-loop control generators for nonlinear systems". Int. J. of Control, 47 (1988), pp. 537-556.

[38] H. J. Sussmann, V. Jurdjevic, "Controllability of Nonlinear systems". J. of Diff. Eq., 12 (1972), pp.95-116.

[39] A.M. Vinogradov : "Local Symmetries and Conservation Laws". Acta Appl. Math., vol. 2 (1984), pp. 21-78.

[40] J.C. Willems, "Paradigms and puzzles in the theory of dynamical systems". IEEE Trans. on Autom. Cont., vol. 36 (1991), pp. 259-294.

[41] W. A. Wolovich, Linear multivariable systems, Springer-Verlag, Applied mathematical sciences, vol. 11, 1974.

${ }^{5}$ ESAIM: Control, Optim. \&3 Calc. of Var., 2 (1997), pp. 151-230, http://www.edpsciences.com/cocv/. 